\title{
$k$-FLAT STRUCTURES AND EXOTIC CHARACTERISTIC CLASSES
}

\author{
LISA R. GOLDBERG
}

\begin{abstract}
We generalize the concept of "foliation" and define $k$-flat structures; these are smooth vector bundles with affine connections whose characteristic forms vanish above a certain dimension. Using semisimplicial techniques we construct a classifying space for $k$-flat structures, and prove a classification theorem for these structures on smooth manifolds.

Techniques from rational homotopy theory are used to relate the exotic characteristic classes of foliations to the rational homotopy groups and cohomology of the classifying space.
\end{abstract}

Introduction. A familiar technique in algebraic topology is to reduce the problem of studying structures on a large collection of objects to the study of a universal example which "contains" all the others. Consider the problem of classifying vector bundles over $\mathrm{CW}$ complexes, in this case we need only look at spaces $B O^{k}$ and $B U^{k}$, and their canonical $k$-plane bundles [9]. A nice feature of this example is that the algebraic invariants of $k$-plane bundles (i.e. Stiefel-Whitney classes, Chern classes, Euler class) are contained in the cohomology of the classifying spaces.

In this paper, we look at a set of invariants which can be attached to foliated smooth manifolds. These are the so-called "exotic characteristic classes" of foliations which were constructed in the late 1960's independently by Bott, Haefliger, Gelfand-Fuks, Kamber-Tondeur, and Malgrange; they can be defined using purely geometric techniques (i.e. see [1] ).

At about the same time, Haefliger [5] developed a topological classification of foliations using $B \Gamma$ spaces, and has applied the theory to smooth manifolds via theorems of Thurston and Gromov-Phillips. One would like to demonstrate a relationship between the exotic characteristic classes and the cohomology of the classifying spaces as in the example above, although this has proven to be difficult. Nevertheless, a lot is known about the homotopy type of $B \Gamma_{k}$, largely through the efforts of Hutsch, Hurder, Kamber-Tondeur, Rasmussen, Thurston, et al.

In $\S 1$, we introduce $k$-flat structures, which generalize the concept of foliations. These are smooth vector bundles with affine connections whose characteristic forms vanish above dimension $2 k$; they constitute the largest classes of objects for which exotic characteristic classes can be defined. $k$-flat structures and exotic characteristic classes were studied by Lehmann in [17].

Using semisimplicial techniques, we will construct classifying spaces for $k$-flat structures and prove a classification theorem for these structures on smooth manifolds. $\S 2$ contains a brief review of semisimplicial methods, while $\S 3$ contains the constructions and proofs.

Received by the editors March 29, 1984 and, in revised form, November 18, 1985.

1980 Mathematics Subject Classification (1985 Revision). Primary 57R30; Secondary 57M40. 
In $\S 4$, we use methods from rational homotopy theory to relate the exotic characteristic classes to the rational homotopy groups and cohomology of the classifying spaces. These methods were introduced by Hurder [6] to study the connection between the homotopy type of $B \Gamma_{k}$ and the exotic classes.

$\S 5$ contains examples and concluding remarks.

This paper is a condensed version of my doctoral dissertation; I would like to thank my advisor Dr. Edgar H. Brown, Jr., for his guidance and support. I would also like to thank Dr. Harold Levine for his mathematical assistance. Finally, I would like to give special thanks to the referee. His (or her) comments and criticisms were immensely helpful. In particular, the referee is responsible for the outline of the example in $\S 5$, and for bringing to my attention the prior work of Lehmann.

1. Let $M$ be a finite dimensional $C^{\infty}$ manifold and let $\varsigma$ be a $C^{\infty} \mathbf{R}^{k}$-vector bundle over $M . C^{\infty}(\varsigma)$ will denote the space of smooth sections of $\varsigma$ with the Whitney $C^{\infty}$ topology, $C^{\infty}(M)$ is the space of smooth real valued functions on $M$, and $T * M$ is the tangent bundle to $M$. Recall that an affine connection on $\zeta$ is an R-linear map $\nabla: C^{\infty}(T * M) \times C^{\infty}(\varsigma) \rightarrow C^{\infty}(\varsigma)$ satisfying

1. $\nabla(f X, s)=f \nabla(X, s)$,

2. $\nabla(X, f s)=X(f)(s)+f \nabla(X, s)$

for $X \in C^{\infty}(T * H), s \in C^{\infty}(\varsigma), f \in C^{\infty}(M)$.

Associated to $\nabla$ is its curvature tensor

$$
K_{\nabla}(X, Y, s)=\nabla(X, \nabla(Y, s))-\nabla(Y, \nabla(X, s))-\nabla([X, y], s) .
$$

We can describe $\nabla$ and $K_{\nabla}$ locally in the following way: Choose a framed open cover $\left\{U_{\alpha}, s_{1}^{\alpha}, \ldots, s_{k}^{\alpha}\right\}$ for $M$, and let $\omega^{\alpha}=\left(\omega_{i j}^{\alpha}\right)$ and $\Omega^{\alpha}=\left(\Omega_{i j}^{\alpha}\right)$ be the $k \times k$ matrices of 1 - and 2 -forms defined by

$$
\nabla\left(X, s_{i}^{\alpha}\right)=\sum_{j} \omega_{i j}^{\alpha}(X) s_{j}^{\alpha}, \quad K_{\nabla}\left(X, Y, s_{i}^{\alpha}\right)=\sum_{j} \Omega_{i j}^{\alpha}(X, Y) s_{j}^{\alpha} .
$$

$\nabla$ and $K_{\nabla}$ are completely determined by these local matrices, and $\omega^{\alpha}$ and $\Omega^{\alpha}$ are related by the identity $\Omega^{\alpha}=d \omega^{\alpha}-\omega^{\alpha} \wedge \omega^{\alpha}$.

All $C^{\infty}$ bundles admit affine connections, and we can define the characteristic classes of a vector bundle using a connection and its curvature tensor via the ChernWeil construction, which we briefly describe below.

Let $I\left(G l_{k}\right)$ be the graded algebra of $G l_{k}$-invariant polynomials $P: G l_{k} \rightarrow \mathbf{R}$. $I_{d}\left(G l_{k}\right)$ is the vector space of polynomials which are homogeneous of degree $d$, and [1] proves that $I\left(G l_{k}\right) \cong\left[p_{1}, p_{2}, \ldots, p_{k}\right]$, where $p_{i}$ has degree $i$.

Suppose $\nabla$ is an affine connection on $\varsigma$ and $\left\{U_{\alpha} ; s_{1}^{\alpha}, \ldots, s_{k}^{\alpha}\right\}$ is a framed open cover for $\nabla$. Let $\Omega^{\alpha}, \omega^{\alpha}$ be as above. Then for any $P \in I_{d}\left(G l_{k}\right)$

$$
P\left(\Omega^{\alpha}\right) / U_{\alpha} \cap U_{\beta}=P\left(\Omega^{\beta}\right) / U_{\alpha} \cap U_{\beta} .
$$

Since $K(f X, g Y)(h s)=f g h K(X, Y)(S)$ for $f, g, h \in C^{\infty}(M)[\mathbf{9}]$, there is a $2 d$-form $P\left(K_{\nabla}\right)$ on $M$ such that $P\left(K_{\nabla}\right) \mid U_{\alpha}=P\left(\Omega^{\alpha}\right)$.

Let $I=\mathbf{R}\left[c_{1}, c_{2}, \ldots\right]$ be the free polynomial algebra on generators $c_{i}$ in dimension $2 i$ with trivial differential, and let $\Omega^{*} M$ denote the $C^{\infty}$ de Rham algebra on $M$. Define a map $\nabla_{*}: I \rightarrow \Omega^{*} M$ by $\nabla_{*} c_{i}=p_{i}\left(K_{\nabla}\right) \in \Omega^{2 i} M$ if $i \leq k$ and $\nabla_{*} c_{i}=0$ if $i>k . \nabla_{*} c_{i}$ is the $i$ th Chern polynomial of $\nabla$. Since $d p_{i}\left(K_{\nabla}\right)=0$ for all $i, \nabla_{*}$ is a chain map and $\left[\nabla_{*} c_{2 i}\right] \in H^{2 i}(M, \mathbf{R})$ is the $i$ th Pontrjagin class of $\varsigma[\mathbf{9}]$. 
If $\zeta$ is equipped with certain types of extra structure, the Chern-Weil construction can be extended to give secondary invariants related to that structure. For example, if $\zeta$ is the normal bundle to a codimension $k$ foliation $\mathscr{T}, \zeta$ admits an adapted connection $\nabla(\mathscr{T})$, which has the property that $K_{\nabla(\mathscr{J})}(X, Y, s)=0$ whenever $X$ and $Y$ are vector fields tangent to $\mathscr{T}[2]$. It follows that $\nabla(\mathscr{T})_{*}$ is the zero map in dimensions greater than $2 k$. Therefore, if $I_{k}$ is the quotient of $I$ by all elements of total degree $>2 k$, then $\nabla(\mathscr{T})_{*}$ induces a chain map from $I_{k}$ to $\Omega^{*} M$. Define a differential graded algebra $W O_{k}=I_{k} \otimes E_{\mathbf{R}}\left(h_{1}, h_{3}, \ldots, h_{e}\right)$ where $h_{i}$ has degree $2 i-1, l$ is the largest odd integer less than or equal to $k$, and $d\left(c_{i} \otimes 1\right)=0$, $d\left(1 \otimes h_{i}\right)=c_{i} \otimes 1$. It is proved in [1] that $\nabla(\mathscr{T})_{*}: I_{k} \rightarrow \Omega^{*} M$ extends to a chain map $\nabla(\mathscr{T})_{*}: W O_{k} \rightarrow \Omega^{*} M$ whose induced map in cohomology depends only on the concordance class of $\mathscr{T}$. The classes in the image $H(\nabla(\mathscr{T}))_{*}\left(H^{*} W O_{k}\right)$ are the exotic characteristic classes of $\mathscr{T}$.

If the normal bundle $\zeta$ of $\mathscr{T}$ is a trivial bundle, the map $\nabla(\mathscr{S})_{*}$ extends to a larger complex: $W_{k}=I_{k} \otimes E_{\mathbf{R}}\left(h_{1}, h_{2}, \ldots, h_{k}\right)$ such that degree $\left(h_{i}\right)=2 i-1$ and $d\left(c_{i} \otimes 1\right)=0, d\left(1 \otimes h_{i}\right)=c_{i} \otimes 1$. As above, the induced map $H \nabla(\mathscr{T})_{*}: H^{*} W_{k} \rightarrow$ $H^{*} M$ depends only on the concordance class of $\mathscr{T}$.

We remark that in both the trivial and nontrivial cases, the definition of the characteristic classes relies only on the adapted connection $\nabla(\mathscr{T})$ and its vanishing property. This suggests the following generalization:

An affine connection $\nabla$ on $\zeta$ is $k$-flat if $\nabla_{*}: I \rightarrow \Omega^{*} M$ is the zero map in dimensions greater than $2 k$. $(\varsigma, \nabla)$ is a $k$-flat structure on $M$ if $\nabla$ is a $k$-flat connection on $\varsigma$. A map of $k$-flat structures $\phi:(\zeta, \nabla) \rightarrow\left(\varsigma^{\prime}, \nabla^{\prime}\right)$ on $M$ is a vector bundle isomorphism $\phi: \varsigma \rightarrow \zeta^{\prime}$ such that, if $s$ and $s^{\prime}$ are sections of $\varsigma$ and $\varsigma^{\prime}$ respectively so that

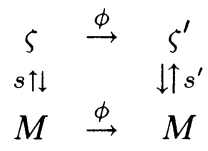

commutes, then

$$
\nabla(X, s)(m)=\nabla^{\prime}\left(Y, s^{\prime}\right)(\phi(m))
$$

where $Y$ is a vector field such that $d \phi(X(m))=Y(\phi(m))$. If $(\varsigma, \nabla)$ is a $k$-flat structure on $M$ and $\zeta$ is a trivial bundle, a trivialization for $(\zeta, \nabla)$ is a map $\phi$ : $\left(M \times \mathbf{R}^{k} \rightarrow M, \nabla^{\prime}\right) \rightarrow(\varsigma, \nabla)$ of $k$-flat structures.

Suppose $f: N \rightarrow M$ is a $C^{\infty}$ map and $(\varsigma, \nabla)$ is a $k$-flat structure on $M$. We can define an affine connection $f^{*} \nabla$ on $f^{*} \zeta$ as follows: Choose a framed open cover $\left\{U_{\alpha} ; s_{1}^{\alpha}, \ldots, s_{k}^{\alpha}\right\}$ for $\zeta$. Then $\left\{f^{-1}\left(U_{\alpha}\right), f^{*} s_{1}^{\alpha}, \ldots, f^{*} s_{k}^{\alpha}\right\}$ is a framed open cover for $f^{*} \varsigma$ and if $X \in C^{\infty}\left(T_{*} f^{-1}(U)\right), y \in f^{-1}(U)$, we define

$$
f^{*} \nabla\left(X, f^{*} s_{i}^{\alpha}\right)(y)=\left(y, \nabla\left(X^{\prime}, s_{i}^{\alpha}\right)\right)(f(y))
$$

where $X^{\prime} \in C^{\infty}\left(T^{*} M\right)$ satisfies $X^{\prime}(f(y))=d f(X(y))$.

If $w^{\alpha}=\left(w_{i j}^{\alpha}\right)$ and $\Omega^{\alpha}=\left(\Omega_{i j}^{\alpha}\right)$, are the connection and curvature matrices of $\nabla$ on $\left\{U^{\alpha} ; s_{1}^{\alpha}, \ldots, s_{k}^{\alpha}\right\}, f^{*} w^{\alpha}$ and $f^{*} \Omega^{\alpha}$ are the connection and curvature matrices of $f^{*} \nabla$ on

$$
\left\{f^{-1}\left(U_{\alpha}\right), f^{*} s_{1}^{\alpha}, \ldots, f^{*} s_{k}^{\alpha}\right\}
$$

Any relations among the $\Omega_{i j}^{\alpha}$ 's must also be satisfied by $f^{*} \Omega_{i j}^{\alpha}$ 's; in particular, $\nabla k$-flat implies $f^{*} \nabla$ is $k$-flat. Therefore, we have an induced $k$-flat structure $f^{*}(\varsigma, \nabla)=\left(f^{*} \varsigma, f^{*} \nabla\right)$ on $N$. 
$k$-flat structures $\left(\varsigma_{0}, \nabla_{0}\right)$ and $\left(\varsigma_{1}, \nabla_{1}\right)$ on $M$ are concordant if there is a $k$-flat structure $(\varsigma, \nabla)$ on $M \times I$ such that $i_{j}^{*}(\varsigma, \nabla)=\left(\varsigma_{j}, \nabla_{j}\right)$ for $j=0,1$. Let $F_{k}(M)$ denote the set of $k$-flat structures on $M$. Concordance is an equivalence relation on $F_{k}(M)$ and we let $c F_{k}(M)=F_{k}(M) / \sim$. If $f: N \rightarrow M$ is as above, the map $f^{*}: F_{k}(M) \rightarrow F_{k}(N)$ preserves concordance classes and induces a map $f^{*}: c F_{k}(M) \rightarrow c F_{k}(N)$. Note that if $(\zeta, \nabla) \sim\left(\zeta^{\prime}, \nabla^{\prime}\right) \in F_{k}(M)$, then $\zeta$ is isomorphic to $\zeta^{\prime}$. Conversely, given a particular vector bundle $\zeta$, we will want to consider the set of concordance classes $\left\{\left[\left(\zeta_{1}^{\prime}, \nabla^{\prime}\right)\right] \in c F_{k}(M) \mid \varsigma^{\prime}\right.$ is isomorphic to $\varsigma\}$, and we denote this set by $c F_{k}(M, \varsigma)$. The extended Chern-Weil construction applies to the set of all $k$-flat structures on $M ;(\varsigma, \nabla) \in F_{k}(M)$ induces a chain map $\nabla^{*}: W O_{k} \rightarrow \Omega^{*} M$ (or $\nabla^{*}: W_{k} \rightarrow \Omega^{*} M$ if $\zeta$ is trivial) whose induced map in cohomology depends only on the concordance class of $(\zeta, \nabla)$.

Our discussion has an analog in the category of $C^{\infty}$ complex vector bundles. The concepts of connection and curvature tensor carry over directly, we just replace the real numbers with the complex numbers throughout. If we let $I, I_{k}, W O_{k}, W_{k}$ also denote their own complexifications, we define the complex Chern-Weil map of a complex connection $\nabla$ on a complex $k$-plane bundle $\gamma \cdot \nabla_{x}$ maps $I$ into the complexified de Rham algebra $\Omega_{\mathrm{C}}^{*} M$, and the classes in the image of the induced map in cohomology are the Chern classes of $\gamma$. Complex $k$-flat structures are defined similarly to real $k$-flat structures, and $F_{k}^{c}(M)$ and $c F_{k}^{c}(M)$ will denote the sets of complex $k$-flat structures, and concordance classes of complex $k$-flat structures, respectively. We note that, as above, $(\gamma, \nabla) \in F_{k}^{c}(M)$ induces a chain map $\nabla_{*}: W O_{k} \rightarrow \Omega_{c}^{k} M$ (or $\nabla_{*}: W_{k} \rightarrow \Omega_{c}^{*} M$ ) whose induced map in cohomology depends only on the concordance class of $(\gamma, \nabla)$.

The main results of this paper can now be stated more precisely. Let $B G l^{k}$ denote the classifying space for $k$-plane bundles [9]. It is well known that a $k$-plane bundle $\xi \stackrel{\pi}{\rightarrow} M$ determines a classifying map $\xi: M \rightarrow B G l^{k}$, and that there is a canonical bijection between homotopy classes of maps $\left[M, B G l^{k}\right]$, and isomorphism classes of $k$-plane bundles over $M$.

THEOREM 3.5. There is a fibration of $C W$ complexes $B \pi^{k}: B C^{k} \rightarrow B G l^{k}$ with the following properties. $B C^{k}$

(i) $A k$-flat structure $(\xi, \nabla)$ determines a lifting of the classifying map of $\xi$ to

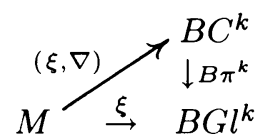

(ii) There is a canonical bijection between concordance classes of $k$-flat structures on $M$ and homotopy classes of maps $\left[M, B C^{k}\right]$.

We are also able to show a relationship between the topology of the fibration $B \pi^{k}: B C^{k} \rightarrow B G l^{k}$ and the cohomology of $W^{k}$. Let $F C^{k}$ denote the homotopy fibre of $B C^{k}$.

Proposition 3.6. $\pi_{i} F C^{k}=0,1 \leq i \leq 2 k$.

THEOREM 4.10. There is a subcomplex $V_{k} \subset W_{k}$ (see $\S 4$ ) such that $i_{k}: H^{*} V_{k}$ $\rightarrow H^{*} W_{k}$ is injective.

Furthermore, there is an injective map $H^{*} V_{k} \rightarrow H^{*} F C_{k}$. 
2. We will carry out our constructions in the category of semisimplicial sets. This section contains standard technical results which we will need. For proofs and further details, please refer to $[\mathbf{8}, \mathbf{1 0}]$.

A pseudogroup $\Gamma$ is a category in which each morphism is invertible and the class of all morphisms forms a set. If $\Gamma_{1}$ and $\Gamma_{2}$ are pseudogroups, a map from $\Gamma_{1}$ to $\Gamma_{2}$ is a functor from $\Gamma_{1}$ to $\Gamma_{2}$. Source and target maps $\sigma$ and $\tau$ are defined in the obvious way, and composition of functions induces a multiplication on the set $\left\{\left(g, g^{\prime}\right) \in \operatorname{mor} \Gamma \times \operatorname{mor} \Gamma \mid \sigma\left(g^{\prime}\right)=\tau(g)\right\}$. If obj $\Gamma$ consists of a single element, $\Gamma$ is a group.

Haefliger [5] extends the theory of group structures and classifying spaces in the topological category to include arbitrary pseudogroups. We will make a similar generalization in a semisimplicial setting.

Let $\Delta_{n}=\left\{\left(t_{0}, t_{1}, \ldots, t_{n}\right) \in R^{n+1} \mid \sum t_{i}=1\right\}$ be the $n$-simplex, and let SS be the category whose objects are $\left\{\Delta_{n} \mid n \geq 0\right\}$ and whose morphisms are affine extensions of order preserving vertex maps. If $C$ is a category (i.e., sets, groups, pseudogroups) a semisimplicial (ss) object in $C$ is a contravariant functor from $\mathrm{SS}$ to $C$, and a map of ss objects is a natural transformation of functors.

Let $\Gamma$ be an ss pseudogroup; then each $\Gamma_{n}$ is a pseudogroup and $\partial_{i}: \Gamma_{n} \rightarrow \Gamma_{n-1}$ and $s_{i}: \Gamma_{n} \rightarrow \Gamma_{n+1}$ are functors satisfying the usual relations. Note that obj $\Gamma$ and mor $\Gamma$ are ss sets and $\sigma, \tau: \operatorname{mor} \Gamma \rightarrow \operatorname{obj} \Gamma$ are ss maps.

Let $\mathscr{S}$ be the category of ss sets and suppose $S \in \mathscr{S}$. A $\Gamma-1$-cochain $\psi$ on $S$ is a collection of ss maps $\psi(\alpha): \tilde{\alpha} \rightarrow \operatorname{mor} \Gamma$ for $\alpha \in S$ such that, for each pair $\alpha, \beta$ and each $t \in \tilde{\alpha} \cap \tilde{\beta}$, there is a $g \in \Gamma$ for which $\sigma(\psi(\alpha)(t))=\sigma(g \circ \psi(\beta)(t))$. $\psi\left(s_{i} \alpha\right)\left(s_{i} \alpha\right)=s_{i}(\psi(\alpha)(\alpha))$ for all degeneracies $s_{i}$.

A $\Gamma-2$-cochain $\phi$ on $S$ is a collection of ss maps $\phi(\alpha, \beta): \tilde{\alpha} \cap \tilde{\beta} \rightarrow \operatorname{mor} \Gamma$ for $\alpha, \beta \in S$ such that if $t \in \tilde{\alpha} \cap \tilde{\beta} \cap \tilde{\gamma}, \sigma \phi(\alpha, \beta)(t)=\tau \phi(\beta, \gamma)(t)$. $\phi$ is a cocycle if $\phi(\alpha, \gamma)(t)=\phi(\alpha, \beta)(t) \circ \phi(\beta, \gamma)(t)$ whenever $t \in \tilde{\alpha} \cap \tilde{\beta} \cap \tilde{\gamma}$. The cocycle condition implies $\phi(\alpha, \alpha)(t)=\operatorname{id}(\sigma \phi(\alpha, \alpha)(t))=\operatorname{id}(\tau \phi(\alpha, \alpha)(t))$ and $\phi(\alpha, \beta)(t)=(\phi(\beta, \alpha)(t))^{-1}$. A cocycle $\phi$ is normalized if $s_{i}(\phi(\alpha, \alpha)(\alpha))=\phi\left(s_{i} \alpha, s_{i} \alpha\right)\left(s_{i} \alpha\right)$ for each degeneracy $s_{i}$.

If $\psi$ is a $\Gamma-1$-cochain and $\phi$ is a $\Gamma$-cocycle such that $\sigma \psi(\alpha)(\alpha)=\tau \phi(\alpha, \alpha)(\alpha)=$ $\sigma \phi(\alpha, \alpha)(\alpha)$, then $\psi \cdot \phi$ is the $\Gamma$-cocycle given by

$$
\psi \cdot \phi(\alpha, \beta)(t)=\psi(\alpha)(t) \circ \phi(\alpha, \beta)(t) \circ \psi(\beta)(t)^{-1} .
$$

Note that $\psi \cdot \phi$ is normalized if both $\psi$ and $\phi$ are normalized, and $\phi_{1} \sim \phi_{2} \leftrightarrow \phi_{1}=$ $\psi \cdot \phi_{2}$ is an equivalence relation.

Let $\hat{\Gamma}(S)$ be the set of normalized $\Gamma$-1-cochains on $S, \bar{\Gamma}(S)=$ set of normalized $\Gamma$-cocycles on $S$ and $\Gamma(S)=\bar{\Gamma}(S) / \sim$. An element of $\Gamma(S)$ is a $\Gamma$-structure on $S$.

If $f: S \rightarrow T$ is an ss map, there are induced maps $\hat{\Gamma}(f): \hat{\Gamma}(T) \rightarrow \hat{\Gamma}(S)$ and $\bar{\Gamma}(f): \bar{\Gamma}(T) \rightarrow \bar{\Gamma}(S)$ defined by

$$
\begin{aligned}
& \hat{\Gamma}(f)(\psi)(\alpha)(t)=\psi(f(\alpha))(f(t)), \\
& \bar{\Gamma}(f)(\phi)(\alpha, \beta)(t)=\phi(f(\alpha), f(\beta))(f(t)) .
\end{aligned}
$$

Since $\bar{\Gamma}(f)(\psi \cdot \phi)=\hat{\Gamma}(f)(\psi) \cdot \bar{\Gamma}(f)(\phi), \bar{\Gamma}(f)$ induces a map $\Gamma(f): \Gamma(T) \rightarrow \Gamma(S)$.

Define an ss set $B \Gamma$ where $B \Gamma_{n}=\bar{\Gamma}\left(\tilde{\Delta}_{n}\right)$ and $\partial_{i}: B \Gamma_{n} \rightarrow B \Gamma_{n-1}$ and $s_{i}: B \Gamma_{n} \rightarrow$ $B \Gamma_{n+1}$ are defined by $\partial_{i} \alpha=\bar{\Gamma}\left(\gamma_{i}\right)(\alpha)$ and $s_{i} \alpha=\bar{\Gamma}\left(p_{i}\right)(\alpha)$. 
PROPOSITION 2.2. There is a $\Gamma$-cocycle $\bar{\omega} \in \bar{\Gamma}(B \Gamma)$ such that the natural transformation $\overline{\mathscr{T}}: \mathscr{P}(S, B \Gamma) \rightarrow \bar{\Gamma}(S)$ defined by $\overline{\mathscr{T}}(f)=\bar{\Gamma}(f)(\bar{\omega})$ is a bijection.

In other words, to each cocycle $\phi \in \bar{\Gamma}(S)$, there is a unique classifying map $\hat{\phi}: S \rightarrow B \Gamma$, such that $\bar{\Gamma}(\hat{\phi})(\bar{\omega})=\phi$. $B \Gamma$ acts as a classifying complex for $\Gamma$ structures on simplicial sets (i.e., ss sets generated by simplicial complexes). Let $\overline{\mathscr{S}} \in \mathscr{P}$ be the subcategory of simplicial sets. If $S \in \overline{\mathscr{S}}$, a subdivision of $S$ is the simplicial set generated by a subdivision of the underlying simplicial complex. Let $|\cdot|: \mathscr{S} \rightarrow \mathrm{CW}$ complexes be the geometric realization functor [8]. Then it follows from the simplicial approximation theorem as proved in [10] that for any $S \in \overline{\mathscr{S}}$ and any homotopy class of maps $[h]$ from $|S|$ to $|B \Gamma|$, there is a subdivision $S^{\prime}$ of $S$ and a semisimplicial map $g: S^{\prime} \rightarrow B \Gamma$ such that $|g| \simeq h:|S|=\left|S^{\prime}\right| \rightarrow|B \Gamma|$.

Suppose $S^{\prime}$ is a subdivision of $S$. $\Gamma$-structures $\Phi \in \Gamma(S)$ and $\Phi^{\prime} \in \Gamma\left(S^{\prime}\right)$ are concordant if there are cocycles $\phi \in \Phi$ and $\phi^{\prime} \in \Phi^{\prime}$ such that $|\hat{\phi}| \simeq\left|\hat{\phi}^{\prime}\right|:|S|$ $\left(=\left|S^{\prime}\right|\right) \rightarrow|B \Gamma|$. Let $c \Gamma(S)=\bigcup_{S^{\prime}}\left\{\Gamma\left(S^{\prime}\right) \mid S^{\prime}\right.$ is a subdivision of $\left.S\right\} / \sim$, where $\sim$ denotes concordance.

The universal $\Gamma$-structure $\omega \in \Gamma(B \Gamma)$ is the $\Gamma$-structure represented by the $\Gamma$ cocycle $\bar{\omega} \in \bar{\Gamma}(B \Gamma)$ constructed in 2.2. Note that if $|f|:|S| \rightarrow|B \Gamma|$ is represented by an ss map $f: S^{\prime} \rightarrow B \Gamma$, the concordance class of $\Gamma(f)(w)$ depends only on the homology class of $|f|$.

THEOREM 2.3. The natural transformation $\mathscr{T}:[|S|,|B \Gamma|] \rightarrow c \Gamma(S)$ defined by $\mathscr{T}([|f|])=[\Gamma(f)(\omega)]$ is a bijection for all $S \in \overline{\mathscr{P}}$.

REMARK. If $B \Gamma$ is a Kan complex [8], we can write a simpler formulation of 2.3 entirely within the category of ss sets. In this case, we call $\Gamma$-structures $\Phi_{0}, \Phi_{1}, \in \Gamma(S)$ concordant if their classifying maps $\hat{\phi}_{0}, \hat{\phi}_{1}: S \rightarrow B \Gamma$ are homotopic. According to [8], this is an equivalence relation on $\Gamma(S)$ and there is a bijection $\Gamma(S) / \sim \leftrightarrow[S, B \Gamma]$.

We describe below a sufficient condition on $\Gamma$ for which $B \Gamma$ is a Kan complex.

An ss pseudogroup $\Gamma$ is $K a n$ if obj $\Gamma$ and mor $\Gamma$ are Kan complexes and both $\sigma, \tau: \operatorname{mor} \Gamma \rightarrow \operatorname{obj} \Gamma$ are Kan fibrations.

THEOREM 2.4. If $\Gamma$ is Kan, B $\Gamma$ is a Kan complex.

COROLlary 2.5. If $\Gamma$ is Kan and $S$ is an ss set, homotopy is an equivalence relation on $\mathscr{P}(S, B \Gamma)$.

The final result we will need is a relative version of Theorem 2.3. Suppose $\Gamma^{1}$ and $\Gamma^{2}$ are ss pseudogroups. A morphism $\pi: \Gamma^{1} \rightarrow \Gamma^{2}$ is a collection of functors $\left\{\pi_{n}: \Gamma_{n}^{1} \rightarrow \Gamma_{n}^{2}\right\}$ commuting with the boundary and degeneracy maps. We call $\pi: \Gamma^{1} \rightarrow \Gamma^{2}$ a Kan map if $\pi: \operatorname{obj} \Gamma^{1} \rightarrow \operatorname{obj} \Gamma^{2}$ is a Kan fibration and if, for each $\gamma \in \operatorname{mor} \Gamma^{2}$ and each $x \in \operatorname{obj} \Gamma^{1}$ such that $\sigma^{2} \gamma=\pi(x)$, there is some $\gamma^{\prime} \in \operatorname{mor} \Gamma^{1}$ such that $\sigma^{2} \gamma=x$ there is some $\gamma^{\prime} \in \operatorname{mor} \Gamma^{1}$ such that $\pi\left(\gamma^{\prime}\right)=\gamma$.

If $\phi$ is a $\Gamma^{1}$-cocycle on $S, \bar{\pi}_{*} \phi$ is the $\Gamma^{2}$-cocycle given by $\left(\bar{\pi}_{*} \phi\right)(\alpha, \beta)(s)=$ $\pi(\phi(\alpha, \beta)(s))$ and if $\psi$ is a $\Gamma^{1}-1$-cochain on $S, \hat{\pi}_{*} \psi \in \Gamma^{2}(S)$ is defined similarly. Note $\bar{\pi}_{*}(\psi \cdot \phi)=\hat{\pi}_{*}(\psi) \cdot \bar{\pi}_{*}(\phi)$; hence, there is an induced map $\pi_{*}: \Gamma^{1}(S) \rightarrow \Gamma^{2}(S)$. Let $E \pi: E \Gamma^{1} \rightarrow E \Gamma^{2}$ and $B \pi: B \Gamma^{1} \rightarrow B \Gamma^{2}$ be the ss maps such that $E \pi_{n}=\hat{\pi}_{n^{*}}$ and $B \pi_{n}=\bar{\pi}_{n^{*}}$.

Note that $\pi_{*}$ preserves the concordance relation. 
THEOREM 2.6. 1. The natural transformations $\mathscr{T}^{\prime}:\left[|S|,\left|B \Gamma^{1}\right|\right] \leftrightarrow c \Gamma^{1}(S)$ restrict to a bijection $[|\hat{\theta}|,|B \pi|] \leftrightarrow c \Gamma^{1}(S, \theta)$.

2. If $\pi: \Gamma^{1} \rightarrow \Gamma^{2}$ is a Kan map, then $B \pi: B \Gamma^{1} \rightarrow B \Gamma^{2}$ is a Kan fibration.

3. It is our aim in this section to construct a classifying space for concordance classes of $k$-flat structures on manifolds. We show that there is a family of $\mathrm{CW}$ complexes $\left|B C^{k}\right|$ for $k \geq 1$ and fibrations $\left|B \pi^{k}\right|:\left|B C^{k}\right| \rightarrow\left|B G l^{k}\right|$ which satisfy the following property. Given a $C^{\infty}$ vector bundle $\xi$ over $M$, there is a one-to-one correspondence between the set of homotopy classes of liftings $\left[\hat{\xi},\left|B \pi^{k}\right|\right]$ and the set of concordance classes $c F_{k}(M, \xi)$

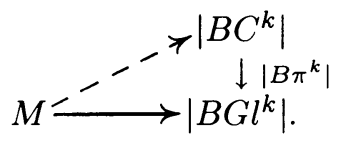

The outline is as follows: we construct a map of ss pseudogroups $\pi^{k}: C^{k} \rightarrow G l^{k}$, where $G l^{k}$ is the ss group adjoint to $\operatorname{Sing}^{\infty} G l^{k}$ (i.e., $G l^{k}$ is the set of $C^{\infty}$ vector bundle isomorphisms $\left\{g: \Delta_{n} \times R^{k} \rightarrow \Delta_{n} \times R^{k}\right\}$ covering the identity map). A $G l^{k}$ structure on a simplicial set $S$ is just a vector bundle on $S$. Theorem 2.6 implies that there is an ss map $B \pi^{k}: B C^{k} \rightarrow B G l^{k}$ such that, given a $G l^{k}$ structure $\bar{\gamma}$ on $S$, there is a bijection between $\left[|\hat{\gamma}|, B \pi^{k}\right]$ and $c C^{k}(S, \bar{\gamma})$.

On the other hand, we show that to each manifold, we can associate a simplicial complex $L$ and a homotopy equivalence $i: M \rightarrow|L|$ which induces a bijection between $c F_{k}(M,|\bar{\gamma}|)$ and $c C^{k}(\widetilde{L}, \bar{\gamma})$. If we choose $\bar{\gamma}$ so that $|\hat{\gamma}|=\hat{\xi}$, the classification theorem is a consequence of the following chain of bijections: $c F_{k}(M, \xi) \leftrightarrow$ $c C^{k}(L, \bar{\gamma}) \leftrightarrow\left[|\hat{\gamma}|,\left|B \pi^{k}\right|\right] \leftrightarrow\left[\hat{\xi},\left|B \pi^{k}\right|\right]$.

Let $\omega \times \mathbf{R}^{k}$ denote the trivial bundle $\omega \times \mathbf{R}^{k} \rightarrow \omega$ for any space $\omega$, and if $\omega^{\prime} \subset \omega$, we identify $\omega^{\prime} \times \mathbf{R}^{k}$ as a subspace of $\omega \times \mathbf{R}^{k}$. Furthermore, if $f: V \rightarrow \omega$ is continuous, $f^{*}\left(\omega \times \mathbf{R}^{k}\right)$ is identified with $V \times \mathbf{R}^{k}$ via $(x, f(x), v) \sim(x, v)$. Finally we assume all bundle maps from $\omega \times \mathbf{R}^{k}$ to itself cover the identity.

A connection on $\Delta_{n} \times \mathbf{R}^{k}$ is an $\mathbf{R}$-linear map $\nabla: C^{\infty}\left(\tau_{*} \Delta_{n}\right) \times C^{\infty}\left(\Delta_{n} \times \mathbf{R}^{k}\right) \rightarrow$ $C^{\infty}\left(\Delta_{n} \times \mathbf{R}^{k}\right)$ satisfying the usual conditions, where $\Delta_{n}$ is viewed as a smooth manifold with corners.

Define an ss set $\theta^{k}$ where $\theta_{n}^{k}=\left\{k\right.$-flat connections on $\left.\Delta_{n} \times \mathbf{R}^{k}\right\}$, and boundaries and degeneracies are given by $\partial_{i} \Delta=\gamma_{i}^{*} \nabla$ and $s_{i} \nabla=\rho_{i}^{*} \nabla$. $G^{k}$ will denote the ss group whose $n$-simplexes are $C^{\infty}$ bundle maps from $\Delta_{n} \times \mathbf{R}^{k}$ to itself and $C^{k}$ is the ss pseudogroup such that obj $C^{k}=\theta^{k}$ and mor $C^{k}=\left\{\left(\nabla, \nabla^{\prime}, g\right) \mid \nabla, \nabla^{\prime} \in \theta^{k}\right.$, $g \in G l^{k}$ and $\left.g^{*} \nabla^{\prime}=\nabla\right\}$. Source and target maps are defined in the obvious way, and there is a map of ss pseudogroups $\pi^{k}: C^{k} \rightarrow G^{k}$ given by $\pi\left(\nabla, \nabla^{\prime}, g\right)=g$.

THEOREM 3.1. The map $\left|B \pi^{k}\right|:\left|B C^{k}\right| \rightarrow\left|B G^{k}\right|$ is a fibration such that, given a simplicial set $S$ and $a G l^{k}$-structure $\overline{\underline{\gamma}} \in G l^{k}(S)$, there is a one-to-one correspondence between $\left[|\hat{\gamma}|,\left|B \pi^{k}\right|\right]$ and $c C^{k}(S, \underline{\bar{\gamma}})$.

PROOF. This is a special case of Theorem 2.6.

Let $h:|L| \rightarrow M$ be a $C^{\infty}$ triangulation of a manifold $M$. For each $\alpha \in L$, let $\tau_{*} \alpha$ be the bundle over $h(|\alpha|)$ of vectors tangent to $h(|\alpha|)$, and if $\xi$ is a $k$-plane bundle over $M, \xi / \alpha$ will denote the restriction of $\xi$ to $h(|\alpha|)$. Note that if $\beta=\partial_{I} \alpha$, there is a restriction map $\partial_{I}: C^{\infty}(\xi / \alpha) \rightarrow C^{\infty}(\xi / \beta)$. 
A singular $k$-flat structure (skf-structure) on $M$ is a collection $\nabla=(h, \xi, \nabla(\alpha))$ such that $h:|L| \rightarrow M$ is a smooth triangulation, $\xi$ is a $C^{\infty}$ bundle over $M$ and, for every $\alpha \in L, \nabla(\alpha)$ is a $k$-flat connection on $\xi / \alpha$ such that

$$
\nabla(\alpha)(v, s)=\left.\nabla(\beta)(v, s)\right|_{h(\beta)}
$$

whenever $\beta=\partial_{I} \alpha$ and $v \in T_{*} \beta$.

If $h(|L|,|K|) \rightarrow(M, \partial M)$ is a relative $C^{\infty}$ triangulation and $\nabla=(h, \xi, \nabla(\alpha))$ is an skf-structure on $M, \nabla / \partial M$ is an skf-structure on $\partial M$, and if $N$ is a component of $\partial M, \nabla / N$ is defined similarly.

Skf-structures $\nabla_{0}$ and $\nabla_{1}$ are concordant if there is an skf-structure $\nabla$ on $M \times I$ such that $\left.\nabla\right|_{M \times\{j\}}=\nabla_{j}$ for $j=0,1$. If $h:|L| \rightarrow M$ is a triangulation, let $S F_{k}(M, h)$ be the set of skf-structures on $M$ with respect to $h$, and if $h^{\prime}:\left|L^{\prime}\right| \rightarrow M$ is a subdivision of $h$ (written $h^{\prime} \leq h$ ), let

$$
c S F_{k}(M, h)=\bigcup_{h^{\prime} \leq h} S F_{k}\left(M, h^{\prime}\right) / \text { concordance. }
$$

A smooth triangulation $h:|L| \rightarrow M$ induces a map $h_{*}: F_{k}(M) \rightarrow S F_{k}(M)$ defined by $h_{*}(\varsigma, \nabla)=(h, \zeta, \nabla(\alpha))$, where $\nabla(\alpha)$ is the restriction of $\nabla$ to $h(|\alpha|)$. $h_{*}$ projects to a map $c F_{k}(M) \rightarrow c S F_{k}(M, h)$, which we also denote by $h_{*}$.

Fix a locally finite, smooth triangulation $h:|L| \rightarrow M$, and let $\tilde{L}$ be the ss set generated by $L$.

LEMMA 3.2. There is a bijection $c S F_{k}(M, h) \leftrightarrow c C^{k}(\tilde{L})$.

PROOF. Let $h^{0}:\left|L^{0}\right| \rightarrow M$ be a subdivision of $h$ and let $\nabla^{0}=\left(h^{0}, \varsigma^{0}, \nabla^{0}(\alpha)\right) \in$ $S F_{k}\left(M, h^{\prime}\right)$ for any $m$-simplex $\alpha$ of $L^{0},\left(\left.\varsigma^{0}\right|_{\alpha}, \nabla^{0}(\alpha)\right)$ is a $k$-flat structure on $h^{0}(|\alpha|)$ so we can choose a trivialization $\bar{\alpha}:\left(\nabla_{n} \times \mathbf{R}^{k}, \bar{\nabla}(\alpha)\right) \rightarrow\left(\left.\varsigma^{0}\right|_{\alpha}, \nabla^{0}(\alpha)\right)$ covering $h^{0} \circ \hat{\alpha}$.

If $\alpha \in L_{m}^{0}, \beta \in L_{p}^{0}, s=\partial_{I} \alpha=\partial_{J} \beta \in \alpha \cap \beta$, let $\phi^{0} \in \bar{C}^{k}\left(L^{0}\right)$ be the cocycle given by

$$
\phi^{0}(\alpha, \beta)(s)=\left(\partial_{I} \bar{\nabla}(\alpha), \partial_{J} \bar{\nabla}(\beta),\left.\hat{\alpha}^{-1} \circ \beta\right|_{\partial_{J} \Delta_{p}}\right) .
$$

If $\phi^{\prime}$ comes from a second choice of trivialization, the change in trivialization produces a 1-chain $\psi$ such that $\phi^{\prime}=\psi \cdot \phi^{0}$, therefore, yields a concordant cocycle; hence, we have a well-defined map $f: \bigcup_{h^{\prime} \leq h} S F_{k}\left(M, h^{\prime}\right) \rightarrow \bigcup_{h^{\prime} \leq h} C^{k}\left(\bar{L}^{\prime}\right)$. Then $f$ projects to a map $F: c S F_{k}(M, h) \rightarrow c C^{k}(\tilde{L})$. If $\nabla^{1} \in S F_{k}\left(M, h^{1}\right)$ is concordant to $\nabla^{0}$, there is an skf-structure $\nabla \in S F_{k}(M \times I, \underline{h})$ such that $\left.\nabla\right|_{M \times\{j\}}=\nabla^{j}$ for $j=0,1$. As above, we define cocycles $\phi \in \bar{C}^{k}(\underline{\tilde{L}}), \phi^{0} \in \bar{C}^{k}\left(\bar{L}^{0}\right), \phi^{1} \in \bar{C}^{k}\left(\tilde{L}^{1}\right)$, so that $|\phi|: M \times I \rightarrow\left|B C^{k}\right|$ is a homotopy between $\left|\phi^{0}\right|$ and $\left|\phi^{1}\right|$. Thus, $f\left(V^{0}\right)$ is concordant to $\Gamma\left(V^{1}\right)$, and $\Gamma:|V| \cdot|\Gamma(V)|$ is well defined. We will show $F$ is a bijection.

Surjectivity. Let $\phi \in \bar{C}^{k}\left(L^{\prime}\right)$ where $h^{\prime}:\left|L^{\prime}\right| \rightarrow M$ subdivides $h$. Consider the $k$ plane bundle $\zeta=\coprod_{\alpha \in L^{\prime}} h^{\prime} \circ \partial\left(\Delta_{n}\right) \times \mathbf{R}^{k} / \sim$ where we identify

$$
\left(h^{\prime} \circ \hat{\alpha}\left(S_{I} x\right), V\right) \sim\left(h^{\prime} \circ \hat{\beta}(x), \pi\left(\phi_{\beta \alpha}(\beta)(x)(V)\right)\right) \quad \forall \beta=\partial_{I^{\alpha}} .
$$

$\varsigma$ car be given a unique smooth structure and we define an skf-structure $\nabla=$ $\left(h^{\prime}, \varsigma, \nabla(\alpha)\right)$ on $M$ as follows: each $\nabla(\alpha)$ is determined by the property that if $f(\alpha): \Delta_{m} \times\left.\mathbf{R}^{k} \rightarrow \zeta\right|_{\alpha}$ is the composite

$$
\Delta_{m} \times \mathbf{R}^{k} \stackrel{h^{\prime} \circ \hat{\alpha} \times 1}{\longrightarrow} h^{\prime} \circ \hat{\alpha}\left(\Delta_{m}\right) \times \mathbf{R}^{k} \stackrel{i}{\rightarrow} \coprod_{\alpha \in L^{\prime}} h^{\prime} \circ \hat{\alpha}\left(\Delta_{m}\right) \times \mathbf{R}^{k} \rightarrow \zeta,
$$


then $f(\alpha):\left(\Delta_{m} \times \mathbf{R}^{k}, \phi(\alpha, \alpha)(\alpha)\right) \rightarrow\left(\left.\varsigma\right|_{\alpha}, \nabla(\alpha)\right)$ is a map of $k$-flat structures. $f([\phi])=\nabla$ by construction, hence $F$ is surjective.

Injectivity. Suppose $\left[\nabla_{0}\right],\left[\nabla_{1}\right] \in c S F_{k}(M, h)$ and $F\left(\left[\nabla_{0}\right]\right)=F\left(\left[\nabla_{1}\right]\right)$. Then we can find cocycle representatives $\phi_{0} \in \bar{C}^{k}\left(\tilde{L}^{0}\right), \phi_{1} \in \bar{C}^{k}\left(\tilde{L}^{1}\right)$ representing $F\left(\left[\nabla_{0}\right]\right)$ and $F\left(\left[\nabla_{1}\right]\right)$, and a homotopy $\psi: M \times I \rightarrow\left|B C^{k}\right|$ between $\left|\phi_{0}\right|$ and $\left|\hat{\phi}_{1}\right|$.

According to $[14$, Theorem 10.6, p. 101], given a bounded manifold $N$ and a smooth triangulation $f:|K| \rightarrow \partial N$, there is a relative smooth triangulation $\underline{f}:(|\underline{K}|,|K|) \rightarrow(N, \partial N)$ extending $f$.

Let $h_{0}:\left|L^{0}\right| \rightarrow M, h_{1}:\left|L^{1}\right| \rightarrow M$ be the subdivisions of $h$ corresponding to $\nabla_{0}$ and $\nabla_{1}$. Choose a smooth triangulation

$$
h:\left(|\underline{L}|,\left|L^{0}\right|,\left|L^{1}\right|\right) \rightarrow(M \times I, M \times\{0\}, M \times\{1\})
$$

extending $h_{0} \cup h_{1}$. By the relative simplicial approximation theorem $[\mathbf{1 0}$, Theorem 5.1$, p. 328$]$, there is a relative subdivision

$$
\underline{h}^{\prime}:\left(\left|\underline{L}^{\prime}\right|,\left|L^{0}\right|,\left|L^{1}\right|\right) \rightarrow(M \times I, M \times\{0\}, M \times\{1\})
$$

and a simplicial map $\theta: \underline{L}^{\prime} \rightarrow B C^{k}$ such that $|\theta| \simeq \gamma$ rel $\partial M \times I$. Therefore, $\left.\theta\right|_{L^{j}}=\hat{\theta}_{j}$ for $j=0,1$.

As above, we construct $\nabla \in S F_{k}\left(M, \underline{h}^{\prime}\right)$ such that $f(\nabla)=\Gamma(\theta)(w) \in C^{k}\left(\underline{\tilde{L}}^{\prime}\right)$. Then $\left.\nabla\right|_{M \times\{j\}}=\nabla_{j}$ for $j=0,1$; hence $\nabla_{0}$ is concordant to $\nabla_{1}$ and $F$ is injective.

To prove Theorem 3.5, we must show that for a fixed triangulation $h$, there is a bijection between the set of concordance classes of skf-structures on $M$ (with respect to $h$ ) and the set of concordance classes of $k$-flat structures on $M$. The bijection is the result of a smoothing procedure which we describe below.

Let $\mathscr{U} \subset M$ be an open set. An skf-structure $\nabla=(h, \xi, \nabla(\alpha)) \in S F_{k}(M, h)$ is smooth on $\mathscr{U}$ if there is a $C^{\infty} k$-flat structure $(\hat{\xi}, \hat{\nabla})$ on $\mathscr{U}$ such that

$$
(\hat{\xi}, \hat{V}) /(\mathscr{U} \cap h|\alpha|)=(\xi / \alpha, \nabla(\alpha) / \mathscr{U} \cap h|\alpha|)
$$

for all $\alpha \in L$. Note that an skf-structure $\nabla$ which is smooth on a neighborhood of the $(n-1)$ skeleton of an $n$-manifold $M$ is smooth on $M$.

In the next lemma, we use an inductive procedure to smooth an skf-structure over the skeleta of a triangulation. We assume first that $M$ is an open subset of Euclidean $n$-space; this enables us to choose a triangulation of $M$ which is compatible with the affine structure of $R^{N}$.

We then construct neighborhoods $\mathscr{U}_{d}$ of the $(d-1)$-skeleton, and smooth maps $G: \mathscr{U}_{d} \rightarrow \mathscr{U}_{d}$ which are the identity near the boundary of $\mathscr{U}_{d}$, and which project smaller neighborhoods $\mathscr{U}_{d}^{\prime} \subset \mathscr{U}_{d}$ onto the $d$-skeleton. We pull back the $k$-flat structure with these maps, the result is a $k$-flat structure which is smooth on a neighborhood of the $d$-skeleton.

LEMMA 3.3. Suppose $M \subset \mathbf{R}^{N}$ is an open subset and $h:|L| \rightarrow M$ is a locally finite smooth triangulation such that $h|\alpha|$ is an affine subset of $\mathbf{R}^{N}$ for all $\alpha$. If $\nabla=(h, \xi, \nabla(\alpha)) \in S F_{k}(M, h)$ and $\nabla$ is smooth on an open neighborhood $\mathscr{U}$ of a subcomplex $h(|K|) \subset M$ such that $h(|K|) \subset \mathscr{U}$, then $\nabla$ is concordant to an skf-structure $\nabla^{\prime}$ which is smooth on $M$ and which agrees with $\nabla$ on some smaller neighborhood $\mathscr{U}^{\prime}$ of $h(|K|)$.

PROOF. We will use an inductive argument to prove an absolute version of 3.3 and then indicate the changes needed for the relative statement. Let $\alpha \in L$. 
Recall:

$$
\begin{aligned}
& \text { St } \alpha=\{\gamma \in L \mid \exists \beta \in L, \alpha \subset \beta, \gamma \subset \beta\}, \\
& \text { Lk } \alpha=\{\beta \in \operatorname{St} \alpha \mid \alpha \not \subset \beta\} .
\end{aligned}
$$

Step 0. If $\varepsilon>0$ and $\alpha \in L$ is a 0-simplex, let $D(\varepsilon, \alpha)=\left\{x \in \mathbf{R}^{N}|| x-h(\alpha) \mid<\right.$ $\varepsilon\}$. For every 0 -simplex $\alpha$, let $\varepsilon(\alpha)>0$ be a number such that $D(3 \varepsilon(\alpha), \alpha) \subset$ $h \mid$ St $\alpha|-h|$ Lk $\alpha \mid$ and if $\beta$ is any other 0-simplex, $\overline{D(3 \varepsilon(\alpha), \alpha)} \cap \overline{D(3 \varepsilon(\beta), \beta)}=\varnothing$. Let $\rho(\alpha): D(\varepsilon(\alpha), \alpha) \rightarrow h|\alpha|$ be the projection map, and $g: I \rightarrow I$ a smooth map that takes a neighborhood of $\partial I$ into itself. Suppose $\rho: \bigcup_{\alpha} D(3 \varepsilon(\alpha), \alpha) \times I \rightarrow I$ is a $C^{\infty}$ map such that

(i) $\rho(m, t)=0$ if $t=0$ or if $|m-h| \alpha|| \geq 2 \varepsilon(\alpha)$,

(ii) $\rho(m, t)=1$ if $g(t)>0$ and $|m-h(\alpha)| \leq g(t) \cdot t(\alpha)$.

1

0
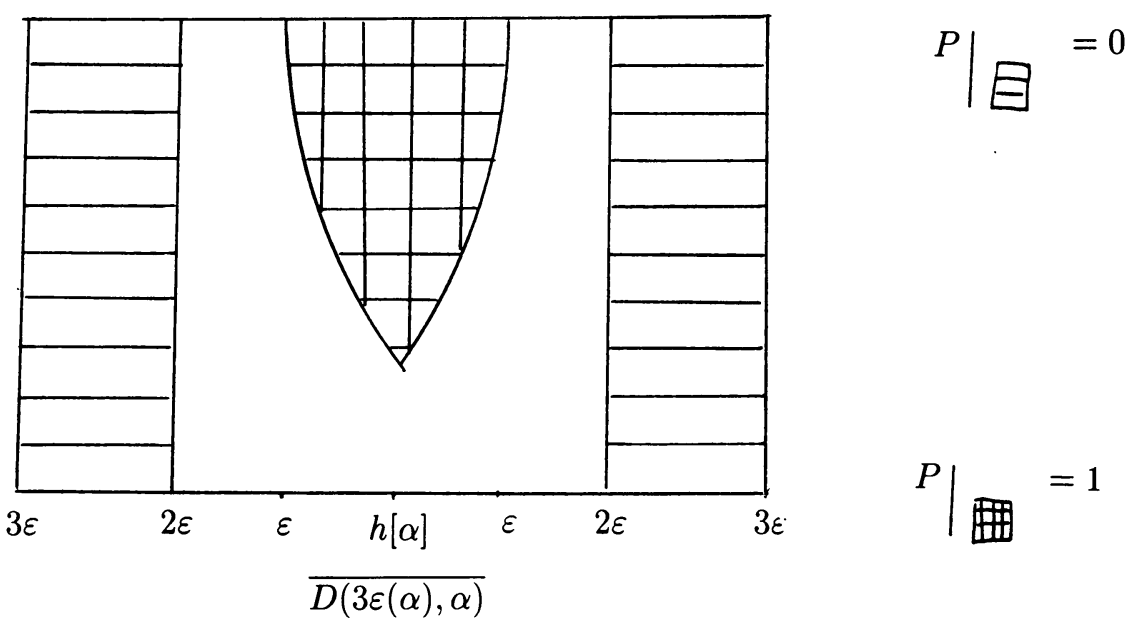

$I$

Let $G: \bigcup_{\alpha} D(3 \varepsilon(\alpha), \alpha) \times I \rightarrow \bigcup_{\alpha} D(3 \varepsilon(\alpha), \alpha)$ be given by

$$
G(m, t)=(1-\rho(m, t)) \cdot m+\rho(m, t) h(\alpha) \text {. }
$$

$G$ is a smooth map and $G / \bigcup_{\alpha} D(3 \varepsilon(\alpha), \alpha)-D(2 \varepsilon(\alpha), \alpha) \times I$ is projection on the first factor. Define $H: M \times I \rightarrow M$ by

$$
\begin{aligned}
H(m, t) & =G(m, t), \quad m \in \bigcup_{\alpha} D(3 \varepsilon(\alpha), \alpha) \\
& =m, \quad m \in M \bigcup_{\alpha} D(2 \varepsilon(\alpha), \alpha) .
\end{aligned}
$$

$H$ is a smooth map since it is given as the union of two smooth maps over an open set. Note that $H /(M \times\{0\})=$ id, $H /(D(\varepsilon(\alpha), \alpha)) \times\{1\}=p(\alpha)$, and for all $\gamma \in L$, $H(h|\gamma| \times I) \subset h|\gamma|$. For suppose $m \in h|\gamma|$; if $m \notin \bigcup_{\alpha}(2 \varepsilon(\alpha), \alpha), H(m, t)=m \in h|\gamma|$ and if $m \in D(2 \varepsilon(\alpha), \alpha)$ for some 0-simplex $\alpha$, then $\alpha<\gamma$ and

$$
H(m, t)=(1-\rho(m, t)) \cdot m+\rho(m, t) \cdot h|\alpha| \in h|\gamma|
$$

by convexity.

Define an skf-structure $H^{*} \nabla=\left(h \times 1, H^{*} \xi, H^{*}(\nabla)(\nu)\right)$ on $M \times I$ as follows: $h \times 1:\left(\left|L \times \Delta_{1}\right|,|L \times\{0\}|,|L \times\{1\}|\right) \rightarrow(M \times I, M \times\{0\}, M \times\{1\})$ is the product triangulation, and if $\nu \in \gamma \times \Delta_{1}$ for some $\gamma \in L$, then $H((h \times 1)|\gamma|) \subset h|\gamma|$ and $H^{*}(\nabla)(\nu)=\left(\left.H\right|_{\nu}\right)^{*}(\nabla(\gamma))$ where $Y$ is any vector field on $M$ tangent to $h|\gamma|$ such that $d H(X(m, t))=Y(H(m, t))$. 
Therefore $\nabla=H^{*} \nabla / M \times\{0\}$ is concordant to $\nabla^{\prime}=H^{*} \nabla / M \times\{1\}$ and $\nabla^{\prime}$ is smooth on $\bigcup_{\alpha} D(\varepsilon(\alpha), \alpha)$ since, for each $\gamma \in L$, and each 0 -simplex $\alpha$,

$$
\left.\left(\xi^{\prime}(\gamma), \nabla^{\prime}(\gamma)\right)\right|_{h|\gamma| \cap D(\varepsilon(\alpha), \alpha)}=\left.p(\alpha)^{*}(\xi / \alpha, \nabla(\alpha))\right|_{h|\gamma| \cap D(\varepsilon(\alpha), \alpha)} .
$$

Inductively assume we have constructed an skf-structure $\nabla^{\prime}=\left(h, \xi^{\prime}, \nabla^{\prime}(\alpha)\right)$ concordant to $\nabla$ which is smooth on a neighborhood $\mathscr{U}$ of the $(d-1)$-skeleton of $h|L|$, and we will suppose that the $(d-1)$-skeleton is a deformation retract of $\mathscr{U}$.

For each $d$-simplex $\alpha$, let $\alpha^{\prime}, \alpha^{\prime \prime}, \alpha^{\prime \prime} \subset h|\alpha|$ be open subsets, each of which is diffeomorphic to the open $d$-ball and so that $\alpha^{\prime} \subset \bar{\alpha}^{\prime} \subset \alpha^{\prime \prime} \subset \bar{\alpha}^{\prime \prime} \subset \alpha^{\prime \prime \prime} \subset \bar{\alpha}^{\prime \prime \prime} \subset$ interior $h|\alpha|$ and $\partial \bar{\alpha}^{\prime}, \partial \bar{\alpha}^{\prime \prime}, \partial \bar{\alpha}^{\prime \prime \prime} \subset \mathscr{U}$.

If $\varepsilon>0$, let $D(\varepsilon)=\left\{x \in \mathbf{R}^{N-d}|| x \mid<\varepsilon\right\}$. Let $V(\alpha)$ be a neighborhood of $\bar{\alpha}^{\prime \prime \prime}$ such that there is a diffeomorphism $\phi(\alpha): \alpha^{\prime \prime \prime} \times D(3) \rightarrow V(\alpha)$ which is the identity in the first factor, and affine in the second factor, and so that

(i) $\overline{V(\alpha)} \cap \overline{V(\beta)}=\varnothing$ if $\alpha \neq \beta$,

(ii) $\overline{V(\alpha)} \subset h|\mathrm{St} \alpha|-h|\mathrm{Lk} \alpha|$.

Let $p(\alpha): V(\alpha) \rightarrow \bar{\alpha}^{\prime \prime \prime}$ be the projection map, and suppose $g: I \rightarrow I$ is a smooth map that takes a neighborhood of $\partial I$ into itself.

Define $C^{\infty}$ maps $\rho_{1}: \bigcup_{\alpha} \bar{\alpha}^{\prime \prime \prime} \rightarrow I$ and $\rho_{2}: D(3) \times I \rightarrow I$ such that

$$
\begin{array}{ll}
\rho_{1}(x)=1 & \text { if } x \in \bigcup_{\alpha} \bar{\alpha}^{\prime}, \\
\rho_{1}(x)=0 & \text { if } x \notin \bigcup_{\alpha} \alpha^{\prime \prime},
\end{array}
$$

$$
\begin{array}{ll}
\rho_{2}(y, t)=0 & \text { if }|y| \geq 2 \text { or if } t=0 \\
\rho_{2}(y, t)=1 & \text { if } g(t)>0 \text { and }|y| \leq t .
\end{array}
$$

Let $G: \bigcup_{\alpha} \bar{\alpha}^{\prime \prime \prime} \times D(3) \times I \rightarrow \bigcup_{\alpha} \bar{\alpha}^{\prime \prime \prime} \times D(3)$ be defined by

$$
G(x, y, t)=\left(x,\left(1-\rho_{2}\left(y, \rho_{1}(x) \cdot t\right)\right) \cdot y\right) .
$$

Note that $G$ is a smooth map, and $G(x, y, t)=(x, y)$ if $t=0$ or $|y| \geq 2$ or $x \in \bigcup_{\alpha} \bar{\alpha}^{\prime \prime \prime}-\alpha^{\prime \prime}$. Let $H: M \times I \rightarrow M$ be defined by

$$
\begin{aligned}
H(m, t) & =m, \quad m \in M \bigcup_{\alpha} \phi(\alpha)^{-1}\left(\alpha^{\prime \prime} \times D(2)\right) \\
& =\phi(\alpha)^{-1}(G(\phi(\alpha)(m, t))), \quad m \in \text { some } V(\alpha) .
\end{aligned}
$$

$H$ is $C^{\infty}$ since it is written as the union over open sets of $C^{\infty}$ maps, and $H / M \times$ $\{0\}=$ id. Furthermore, if $\gamma \in L, H(h|\gamma| \times I) \subset h|\gamma|$; for suppose $m \in h|\gamma|$; then if $m \notin \bigcup_{\alpha} V(\alpha)$, we have $H(m, t)=m \in h|\gamma|$, and if $m \in$ some $V(\alpha)$, then $p(\alpha)(m) \in h|\gamma|$, and if $\phi(\alpha)(m)=(x, y) \in \bar{\alpha}^{\prime \prime \prime} \times D(3)$, then

$$
\begin{aligned}
H(m, t)= & \phi(\alpha)^{-1}\left(x,\left(1-\rho_{2}\left(y, \rho_{1}(x) \cdot t\right)\right) y\right) \\
= & \phi(\alpha)^{-1}\left(x,\left(1-\rho_{2}\left(y, \rho_{1}(x, t)\right) \cdot y+\rho_{2}\left(y, \rho_{1}(x) \cdot \varepsilon\right)\right) \cdot 0\right) \\
= & \left(1-\rho_{2}\left(y, \rho_{1}(x) t\right)\right) \phi(\alpha)^{-1}(x, y) \\
& +\rho_{2}\left(y, \rho_{1}(x) t\right) \phi(\alpha)^{-1}(x, 0) \\
= & \left(1-\rho_{2}\left(y, \rho_{1}(x) t\right)\right) \cdot m+\rho_{2}\left(y, \rho_{1}(x) t\right) \cdot p(\alpha)(m)
\end{aligned}
$$

since $\phi(\alpha)$ is affine. Therefore, $H(m, t) \in h|\gamma|$ by convexity. 
Let $H^{*} \nabla^{\prime}$ be the induced skf-structure defined as in Step 0 above; then $\nabla^{\prime}=$ $H^{*} \nabla^{\prime} / M \times\{0\}$ is concordant to $H^{*} \nabla^{\prime} / M \times\{1\}=\nabla^{\prime \prime}$. We show $\nabla^{\prime \prime}$ is smooth on a neighborhood of the $d$-skeleton of $M$.

Let $H_{1}=H(\cdot, 1): M \rightarrow M$; then $\nabla^{\prime \prime}=H_{1}^{*} \nabla^{\prime}$ and by hypothesis, there is a neighborhood $\mathscr{U}$ and a deformation retraction $r: \mathscr{U} \rightarrow(d-1)$-skeleton, such that

$\nabla^{\prime}$ is smooth on $\mathscr{U}$. Since $\bigcup_{\alpha} \partial \bar{\alpha}^{\prime} \subset \mathscr{U}$, it follows that $\bigcup_{\alpha} h|\alpha|-\alpha^{\prime} \subset \mathscr{U}$, and if $m \in \bigcup_{\alpha} h|\alpha|-\alpha^{\prime}$ then $H_{1}(m)=m$ so by continuity, there is a neighborhood $\mathscr{U}^{\prime} \subset \mathscr{U}$ such that $\bigcup_{\alpha} h|\alpha|-\alpha^{\prime} \subset \mathscr{U}^{\prime}$ and $H_{1}(\mathscr{U}) \subset \mathscr{U} . H_{1}$ is a smooth map; therefore $H_{1}^{*} \nabla^{\prime}=\nabla^{\prime \prime}$ must be smooth on $\mathscr{U}^{\prime}$.

Finally, let $\mathscr{U}(\alpha)=\phi(\alpha)^{-1}\left(\alpha^{\prime} \times D(1)\right)$ for each $\alpha$. Note that $H_{1} \mid \mathscr{U}(\alpha)=$ $p(\alpha) / \mathscr{U}(\alpha): \mathscr{U}(\alpha) \rightarrow \alpha^{\prime}$ and for all $\gamma \in L$,

$$
\left(\xi^{\prime \prime}(\gamma), \nabla^{\prime \prime}(\gamma)\right) / h|\gamma| \cap \mathscr{U}(\alpha)=p(\alpha)^{*}\left(\xi^{\prime}(\alpha), \nabla^{\prime}(\alpha)\right) / h|\gamma| \cap \mathscr{U}(\alpha)
$$

and $\bigcup_{\alpha} p(\alpha)^{*}\left(\xi^{\prime}(\alpha), \nabla^{\prime}(\alpha)\right) / \mathscr{U}(\alpha)$ is a $C^{\infty} k$-flat structure on $\bigcup_{\alpha} \mathscr{U}(\alpha)$. Therefore, $\nabla^{\prime \prime}$ is smooth on $\mathscr{U}^{\prime} \cup \bigcup_{\alpha}(\alpha)$.

If $m \in d$-skeleton, then either $m \in \alpha^{\prime} \subset \mathscr{U}(\alpha)$ for some $\alpha$ or

$$
m \in\left(d \text {-skeleton } \backslash \bigcup_{\alpha} \alpha^{\prime}\right) \subset \mathscr{U} .
$$

Therefore, $\mathscr{U} \cup \bigcup_{\alpha}(\alpha)$ is a neighborhood of the $d$-skeleton.

This completes the inductive step and we conclude that $\nabla$ is concordant to a skf-structure $\nabla^{\prime}$ which is smooth on a neighborhood of the $(N-1)$-skeleton of $M$ and hence, is smooth on $M$.

To prove the relative statement, assume $\nabla$ is already smooth on a neighborhood $\mathscr{U}$ of a subcomplex $h|K|$ of $M$ such that $h|K| \subset \overline{h|K|} \subset \mathscr{U}$. Subdivide the triangulation so that there is a subcomplex $K^{\prime}$ and an open set $\mathscr{U}^{\prime}$ such that $h|K| \subset \mathscr{U}^{\prime} \subset h\left|K^{\prime}\right| \subset \mathscr{U}$ and perform the same induction considering only simplexes outside of $\mathscr{U}^{\prime}$. Note that the construction implies that the concordance restricted to $\mathscr{U}^{\prime} \times I$ is the product concordance.

COROLlaRY 3.4. If $M \subset \mathbf{R}^{N}$ is an open subset, $h:|L| \rightarrow M$ is a triangulation satisfying the hypothesis of 3.3 , and $\xi$ is a $C^{\infty} k$-plane bundle over $M$, there is a bijection $c C^{k}(\tilde{L}, \bar{\gamma}) \leftrightarrow c F_{k}(M, \xi)$ for $\bar{\gamma} \in G^{k}(\tilde{L})$ such that $|\underline{\bar{\gamma}}| \sim h^{*} \xi$. This is a direct consequence of 3.2 and 3.3 .

We define a transformation of functors $\Phi:\left[\circ,\left|B C^{k}\right|\right] \rightarrow c F_{k}(\cdot)$ as follows: If $M \subset \mathbf{R}^{N}$ is open, choose a triangulation $h:|L| \rightarrow M$ satisfying the hypotheses of 3.3 , then $\Phi$ is defined to be the composite of maps

$$
\left[M,\left|B C^{k}\right|\right] \stackrel{h^{*}}{\rightarrow}\left[|L|\left|B C^{k}\right|\right] \stackrel{\int}{\rightarrow} c C^{k}(\tilde{L}) \stackrel{F^{-1}}{\rightarrow} c S F_{k}(M, h) \stackrel{h^{*-1}}{\rightarrow} c F_{k}(M)
$$

constructed in $3.1,3.2$ and 3.4.

For arbitrary $M$, choose an embedding $i: M \rightarrow \mathbf{R}^{N}$ for $N>2 \operatorname{dim}(M)$, and a tubular neighborhood $p: U \rightarrow M$ of $M$ in $\mathbf{R}^{N}$. If we triangulate $U$ by $h:|L| \rightarrow U$ as above, $\Phi$ is the composite

$$
\left[M,\left|B C^{k}\right|\right] \stackrel{P^{*}}{\rightarrow}\left[U,\left|B C^{k}\right|\right] \stackrel{\Phi}{\rightarrow} c F_{k}(U) \stackrel{i^{*}}{\rightarrow} c F_{k}(M) .
$$


THEOREM 3.5. (i) $\Phi$ is a bijection and a natural transformation.

(ii) Let $\left|B \pi^{k}\right|:\left|B C^{k}\right| \rightarrow\left|B G^{k}\right|$ be as in 3.1. If $\varsigma \rightarrow M$ is a smooth $\mathbf{R}^{k}$ vector bundle, $\Phi$ restricts to a bijection $\left[\zeta,\left|B \pi^{k}\right|\right] \leftrightarrow c F_{k}(M, \varsigma)$.

ProOF. (i) If $M \subset \mathbf{R}^{N}$ is open, $\Phi:\left[M,\left|B C^{k}\right|\right] \rightarrow c F_{k}(M)$ is a bijection by 3.1 and 3.4. In general, note that $p^{*}:\left[M,\left|B C^{k}\right|\right] \rightarrow\left[U,\left|B C^{k}\right|\right]$ is an isomorphism, and since $p \circ i=\mathrm{id}: M \rightarrow M$ and $i \circ p: U \rightarrow U$ is smoothly homotopic to id, there is a bijection $i^{*}: c F_{k}(U) \rightarrow c F_{k}(M)$. $\Phi$ does not depend on the choice of embedding or triangulation. Suppose we have embeddings $i_{0}: M \rightarrow \mathbf{R}^{N}, i_{1}: M \rightarrow \mathbf{R}^{P}$, tubular neighborhoods $p_{0}: U_{0} \rightarrow i_{0}(M)$ and $p_{1}: U_{1} \rightarrow i_{1}(M)$, and affine triangulations $h_{0}:\left|L_{0}\right| \rightarrow U_{0}$ and $h_{1}:\left|L_{1}\right| \rightarrow U_{1}$. Without loss of generality we will assume $N=P$.

Choose a one parameter family of embeddings $i_{t}: M \rightarrow R^{N}$ and a one parameter family of tubular neighborhoods $p_{t}: U_{t} \rightarrow i_{t}(M)$ depending smoothly on $t$, connecting $p_{0}: U_{0} \rightarrow i_{0}(M)$ and $p_{1}: U_{1} \rightarrow i_{1}(M)$, and so that $\bigcup_{t} U_{t} \in R^{N+1}$ is an open submanifold. We choose an affine relative triangulation $h:\left(\underline{L}, L_{0}, L_{1}\right) \rightarrow$ $\left(U, U_{0}, U_{1}\right)$.

The following commutative diagram shows that $\Phi$ is independent of the choices indicated:

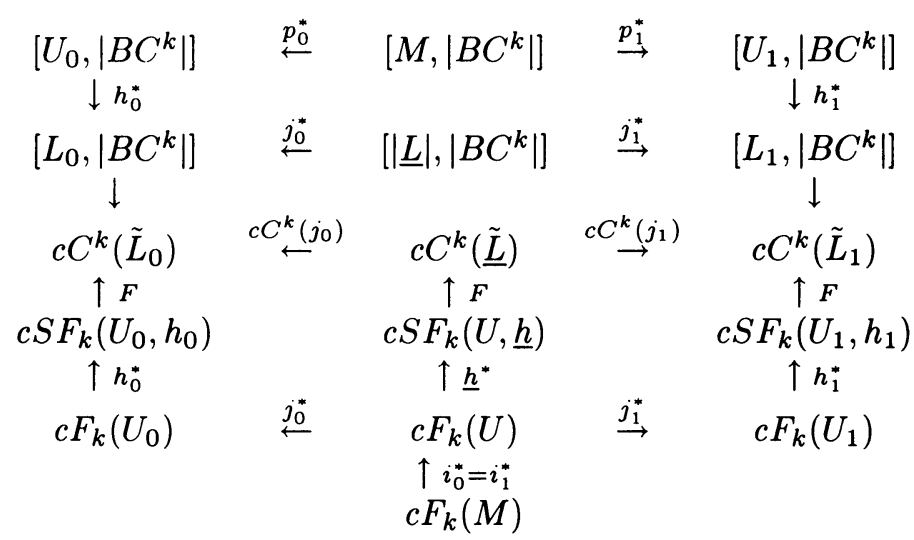

where $j_{t}: U_{t} \rightarrow U$ denotes the inclusion.

To show $\Phi$ is natural, consider first the case where $M$ and $N$ are open subsets of Euclidean space. Let $\alpha: M \rightarrow N$ be a smooth map representing $[\alpha] \in[M, N]$; choose affine triangulation $h_{0}:\left|L_{0}\right| \rightarrow M, h_{1}:\left|L_{1}\right| \rightarrow N$ and a simplicial approximation $g: L_{0} \rightarrow L_{1}$ to $\alpha$.

We will show that the following diagram commutes:

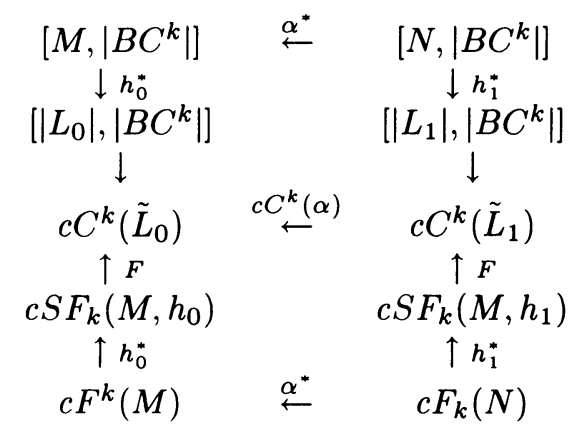


The top square commutes by Theorem 3.1. To show that the bottom square commutes, let $(\zeta, \nabla) \in F_{k}(N)$. We show that $h_{0}^{*} \alpha^{*}(\zeta, \nabla)$ represents the concordance class $F^{-1} \circ c C^{k}(\alpha) \circ F \circ h_{1}^{*}[(\varsigma, \nabla)]$. The latter class is represented by $|g|^{*} \nabla=$ $\left(h_{0},|g|^{*} \xi,|g|^{*} \nabla(\alpha)\right), \alpha \in L_{0}$, where $\nabla=h_{1 *}(\xi, \nabla) \in S F_{*}\left(N, h_{1}\right)$.

Let $H: M \times I \rightarrow N$ be the homotopy between $\gamma$ and $|g|$ such that for any simplex $\alpha \in L_{0},\left.H\right|_{|\alpha| \times I}$ is linear. Therefore, $H^{*} \nabla=\left(h_{0} \times \mathrm{id}, H^{*} \xi, H^{*} \nabla(\nu)\right)$ is an skfstructure on $H \times I$ with respect to the product triangulation, giving a concordance between $|g|^{*} \nabla$ and $h_{0 *} \circ \gamma^{*}(\xi, \nabla)$. Hence $\Phi$ is natural in this case, and the general case follows directly.

(ii) The relative statement for an open subset $M \subset \mathbf{R}^{N}$ is a consequence of 3.1 and 3.4. In general, given a smooth $\xi \rightarrow M$, an embedding $i: M \rightarrow \mathbf{R}^{N}$ and a tubular neighborhood $p: U \rightarrow M$ of $M$ in $\mathbf{R}^{N}$, there are bijections $\left[\hat{\xi},\left|B \pi^{k}\right|\right] \leftrightarrow$ $\left[p^{*} \xi,\left|B \pi^{k}\right|\right]$ and $c F_{k}(M, \xi) \leftrightarrow c F_{k}\left(U, p^{*} \xi\right)$. Hence, the theorem holds for all manifolds.

Hereafter, we will omit the geometric realization symbols and let $B \Pi^{k} \rightarrow B G l_{k}$ denote the fibration of CW complexes. Let $F C^{k}$ be the homotopy theoretic fibre of $B \Pi^{k}$. $F C^{k}$ acts as a classifying space for $k$-flat structures whose underlying bundle is trivialized.

PROPOSITION 3.6. $\Pi_{i} F C^{k}=0$ if $1 \leq i \leq 2 k$.

PROOF. Let $f: S^{n} \rightarrow F C^{k}$ be a continuous map. Up to homotopy, $f$ classifies a $k$-flat structure $\left(S^{n} \times \mathbf{R}^{k}, \nabla\right) . S^{n} \times \mathbf{R}^{k}$ sits inside $D^{n+1} \times \mathbf{R}^{k}$ and we extend $\nabla$ to an affine connection $\widetilde{\nabla}$ on $D^{n+1} \times \mathbf{R}^{k}$ using a partition of unity.

If $P: G l_{k} \rightarrow \mathbf{R}$ is an invariant polynomial (see $\S 1$ ) which is homogeneous of degree $l>k, P\left(K_{\nabla}\right)$ is a $2 l$ form on $D^{n+1}$; therefore $P\left(k_{\nabla}\right)$ is identically zero if $n \leq 2 k$ since $2 l>2 k+1 \geq n+1$.

Hence, $\tilde{\nabla}$ is $k$-flat, and $f$ extends over $D^{n+1}$.

COROLlaRY 3.7. $B \Pi_{*}^{k}: \Pi_{i} B C^{k} \rightarrow \Pi_{i} B G l_{k}$ is an isomorphism if $0 \leq i \leq 2 k$.

LEMMA 3.8. $H B \Pi^{k}: H^{i}\left(B G l_{k}, \mathbf{R}\right) \rightarrow H^{i}\left(B C^{k}, \mathbf{R}\right)$ is the zero map if $i>2 k$.

PROOF. Suppose the converse. Then there is a nonzero Pontrjagin class $c \in$ $H^{i}\left(B C^{k}, \mathbf{R}\right)$. Let $L$ be a finite polyhedron and $f: L \rightarrow B C^{k}$ a continuous map such that $H f(c) \neq 0 \in H^{i}(L, \mathbf{R})$.

Choose an open manifold $\mathscr{U}$, and a homotopy equivalence $j: \mathscr{U} \rightarrow L$. Theorem 3.5 implies that $f \circ j$ is homotopic to a map $g: \mathscr{U} \rightarrow B C^{k}$ that classifies a smooth $k$-flat structure $(\xi, \nabla)$ on $\mathscr{U}$. Therefore, $g^{*}(c) \neq 0 \in \operatorname{Pont}(\xi) \subset H^{*}(\mathscr{U}, \mathbf{R})$, contradicting the $k$-flatness of $\nabla$.

In $\S 4$, we will consider complex vector bundles as well as real bundles, and the methods developed in this section apply directly to this case.

Define an ss set $\theta_{\mathbf{C}}^{k}$ such that $\left(\theta_{\mathbf{C}}^{k}\right)_{n}=\left\{\right.$ complex $k$-flat connections on $\left.\Delta_{n} \times \mathbf{C}^{k}\right\}$, and boundaries and degeneracies are defined by $\partial_{i} \nabla=\gamma_{i}^{*} \nabla$ and $s_{i} \nabla=\rho_{i}^{*} \nabla$. $\theta G_{\mathbf{C}}^{k}$ will be the ss group whose $n$-simplexes are $C^{\infty}$ bundle maps from $\Delta_{n} \times \mathbf{C}^{k}$ to itself, and $C_{\mathbf{C}}^{k}$ is the ss pseudogroup such that obj $C_{\mathbf{C}}^{k}=\theta_{\mathbf{C}}^{k}$ and $\operatorname{mor} C_{\mathbf{C}}^{k}=$ $\left\{\left(\nabla, \nabla^{\prime}, g\right) \mid \nabla, \nabla^{\prime} \in \theta_{\mathbf{C}}^{k}, g \in G_{\mathbf{C}}^{k}\right.$ and $\left.g^{*} \nabla^{\prime}=\nabla\right\}$. Let $\left(\Pi_{\mathbf{C}}^{k}\right): C_{\mathbf{C}}^{k} \rightarrow G_{\mathbf{C}}^{k}$ be the map of ss pseudogroups defined: $\Pi_{\mathbf{C}}^{k}\left(\nabla, \nabla^{\prime}, g\right)=g$. 
THEOREM 3.9. (i) There is a fibration of $C W$ complexes $B \Pi_{\mathbf{C}}^{k}: B C_{\mathbf{C}}^{k} \rightarrow B G_{\mathbf{C}}^{k}$ $(=B G l(k, \mathbf{C}))$ such that, given a $C^{\infty} k$-plane bundle $\xi$ over $M$, there is a bijection $\left[\hat{\xi}, B \Pi_{\mathbf{C}}^{k}\right] \leftrightarrow c F_{k}^{\mathbf{C}}(M, \xi)$.

(ii) Let $F C_{\mathbf{C}}^{k}$ denote the homotopy fibre of $B \Pi_{\mathbf{C}}^{k}$. Then $\Pi_{i} F C_{\mathbf{C}}^{k}=0$ if $1 \leq i \leq 2 k$.

(iii) $H B \Pi_{\mathbf{C}}^{k}: H^{i}\left(B G_{\mathbf{C}}^{k}, \mathbf{C}\right) \rightarrow H^{i}\left(B C_{\mathbf{C}}^{k}, \mathbf{C}\right)$ is the zero map if $i>2 k$.

4. In this section we relate the characteristic classes of foliations to the classifying space for $k$-flat structures constructed in $\S 4$. Following [6], we reformulate the exotic classes as dual homotopy invariants. Techniques from rational homotopy theory are applied to show these invariants can be realized as dual homotopy classes of $B C_{k}$ and $B C_{k}^{\mathbf{C}}$, or equivalently, as cohomology classes of $F C_{k}$ and $F C_{k}^{\mathbf{C}}$.

We begin by recalling some basic facts about homotopy theory and differential graded $(\mathrm{dg})$ algebras. $[\mathbf{1 1}]$ and $[\mathbf{4}]$ will be our references.

Let $\mathbf{F}$ denote either the real or complex numbers. A (graded) algebra over $\mathbf{F}$ is a nonnegatively graded $\mathbf{F}$-module $X$ together with a skew-commutative multiplication $\mu: X \otimes X \rightarrow X$ and a unit $\eta: \mathbf{F} \rightarrow X$. If $x \in X^{m}$ and $y \in X^{n}$, we write $x \cdot y$ for $\mu(X \otimes Y) \in X^{m+n} .(X, d)$ is a dg algebra if $d: X \rightarrow X$ is a degree 1 map of graded F-modules satisfying $d^{2}=0$ and $d(x \cdot y)=d x \cdot y+(-1)^{|x|} x \cdot d y$. When there is no chance of confusion, we let $X$ denote the $d g$ algebra $(X, d)$. $\mathbf{F}$ can be regarded as a dg algebra which is nonzero only in dimension zero, with trivial differential.

An augmentation for $X$ is a dg algebra map $\varepsilon: X \rightarrow \mathbf{F}, \varepsilon h=$ id and $\bar{X}$ will denote the augmentation ideal. Let $\mathscr{A}$ and $\mathscr{A}_{0}$ be the categories of dg and dga (differential graded augmented) algebras respectively.

If $X \in \mathscr{A}$, the cohomology of $X$ is its homology with respect to $d . X$ is connected if $H \eta: H^{0}(\mathbf{F}) \rightarrow H^{0}(X)$ is an isomorphism and $X$ is simply connected if $H^{1}(X)=0$. A connected algebra has a unique augmentation which will be used implicitly.

We briefly describe the homotopy theory of dg algebras. Let $X, Y \in \mathscr{A}, \mathscr{A}(X, Y)$ will denote the set of dg algebra maps from $X$ to $Y$ and $X \otimes Y$ is the usual tensor product of dg algebras.

Let $\Delta(1, *)$ be the dg algebra generated by indeterminates $\tau_{0}$ and $\tau_{1}$ in grade 0 , $d \tau_{0}$ and $d \tau_{1}$ in grade 1 , satisfying relations $\tau_{0}+\tau_{1}=1$, and $d\left(\tau_{i}\right)=d \tau_{i}$ for $i=0,1$.

Maps $f_{0}, f_{1} \in(X, Y)$ are homotopic if there is a dg algebra map $F: X \rightarrow Y \otimes$ $\Delta(1, *)$ such that $\left(1 \otimes \partial_{1}\right) \circ F=f_{i}: X \rightarrow Y$ for $i=0,1$. Homotopy is not generally an equivalence relation on $\mathscr{A}(X, Y)$.

For each $n \geq 0$, let $X(n)=X$ be the sub-dg algebra generated by $\bigcup_{i=0}^{n} X^{i}$ and $d X^{n}$. Inductively define subalgebras $X(n, q)$ for $q \geq 0$ as follows: Let $X(n, 0)=$ $X(n-1)$ and if $q>0$, let $X(n, q)$ be the subalgebra generated by $X(n, q-1)$ and $\left\{x \in X^{n} \mid d x \in X(n, q-1)\right\}$.

We say that an algebra $M \in \mathscr{A}$ is minimal if $M$ is connected, free, and $M(n)=$ $\bigcup_{q \geq 0} M(n, q)$ for all $n$. A minimal model for a connected algebra $X \in \mathscr{A}$ is a map $f: \mu X \rightarrow X$ such that $\mu X$ is a minimal algebra and $f$ is a cohomology isomorphism.

Minimal models are useful because they often simplify calculations. Fortunately, many algebras can be approximated by minimal algebras.

LEMMA 4.1. Every connected algebra $X \in \mathscr{A}$ has a minimal model $f: \mu X \rightarrow X$ and $\mu X$ is unique up to isomorphism [4]. 
The homotopy type of a connected algebra $X \in \mathscr{A}$ is defined to be the isomorphism class of any minimal model $f: \mu X \rightarrow X$ and the dual homotopy groups $\Pi^{n} X$, $n \geq 0$, are the homology groups of the cochain complex $\mu X / \overline{\mu X} \cdot \overline{\mu X}$.

If $M \in \mathscr{A}$ is minimal, the homotopy relation on the set of maps from $M$ to $Y$ is an equivalence relation for all $Y \in \mathscr{A}[\mathbf{4}]$ and we let $[\mu, Y]$ denote the set of homotopy classes of maps from $M$ to $Y$.

The following results and calculations will be needed later on:

LEMMA 4.2. (i) If $g: X \rightarrow Y$ is a cohomology isomorphism and $M$ is minimal, $g:[M, X] \rightarrow[M, Y]$ is a bijection $[\mathbf{4}, 5.7,5.8]$.

(ii) If $X, Y \in \mathscr{A}$ are simply connected and $H g: H^{*} X \rightarrow H^{*} Y$ is an isomorphism, then $\Pi g: \Pi^{*} X \rightarrow \Pi^{*} Y$ is an isomorphism [4].

There is a close relationship between topological homotopy type and algebraic homotopy type. For any finite CW complex or manifold $L$, let

$$
\Pi^{*} L=\operatorname{Hom}\left(\left(\Pi_{*} L\right), \mathbf{R}\right)
$$

and if $L$ is an infinite CW complex, then $\Pi^{*} L=\lim _{\alpha} \Pi^{*} L_{\alpha}$ as $L_{\alpha}$ ranges over all finite subcomplexes of $L$.

THEOREM 4.3. If $M$ is a manifold with $\pi_{1}(M)$ nilpotent and $\Omega_{\mathbf{F}}^{*}(M)$ is its de Rham algebra over $\mathbf{F}$, there is a natural isomorphism $\Pi^{*} \Omega_{\mathbf{F}}^{*}(M) \rightarrow \Pi^{*} M[\mathbf{1 1}]$.

For more general results along these lines, see $[\mathbf{4}, \mathbf{1 1}]$.

Calculations of rational homotopy groups with minimal models is interesting and instructive. The reader is referred to [15, Chapter 13] for further details. We include below a statement of results which we will need later on.

THEOREM 4.4 .

$$
\begin{aligned}
& \Pi^{i} B O^{2 r}=\mathbf{F}, i=0,4, \ldots, 4(r-1), 2 r \\
&=0, \text { all other } i, \\
& \Pi^{i} B O^{2 r+1}=\mathbf{F}, \quad i=0,4, \ldots, 4 r, 2 r \\
&=0, \quad \text { all other } i \\
& \Pi^{i} B U^{n}=\mathbf{F}, \quad i=0,2, \ldots, 2 n, \\
&=0, \quad \text { all other } i[\mathbf{6}] .
\end{aligned}
$$

Recall from $\S 1$, the dg algebra $W_{k}=I_{k} \otimes E_{\mathbf{F}}\left(h_{1}, h_{2}, \ldots h_{k}\right)$ where

$$
I_{k} \cong P_{\mathbf{F}}^{2 k}\left(c_{1}, c_{2}, \ldots, c_{k}\right) \text {. }
$$

Let $J_{k} \subset I_{k}$ be the sub-dg algebra $P_{\mathbf{F}}^{2 k}\left(c_{2}, c_{4}, \ldots, c_{l}\right)$ where $l$ is the largest even integer less than $k$, and let $V_{k}=J_{k} \otimes E_{\mathbf{F}}\left(h_{2}, h_{4}, \ldots, h_{l}\right)$ where $d h_{i}=c_{i}$ and $d c_{i}=0$. Let $\tilde{I}_{k}$ and $\tilde{J}_{k}$ denote the underlying graded $\mathbf{F}$-modules of $I_{k}$ and $J_{k}$ respectively.

We can regard $H^{*} W_{k}$ and $H^{*} V_{k}$ as dg algebras with trivial differential. In both cases all products are zero [6]. Let $\mathscr{L}\left(H^{*} W_{k}\right)$ and $\mathscr{L}\left(H^{*} V_{k}\right)$ be the free graded Lie algebras [6] on $H^{*} W_{k}$ and $H^{*} V_{k}$ respectively. 
THEOREM 4.5 . (i) $\Pi^{*} W_{k} \cong \operatorname{Hom}\left(\mathscr{L}\left(H^{*} W_{k}\right), \mathbf{F}\right)$,

(ii) $\Pi^{*} V_{k} \cong \operatorname{Hom}\left(\mathscr{L}\left(H^{*} V_{k}\right), \mathbf{F}\right)$,

(iii) $\Pi^{*} I_{k} \cong \Pi^{*} W_{k} \oplus \tilde{I}_{k}$,

(iv) $\Pi^{*} J_{k} \cong \Pi^{*} V_{k} \oplus \tilde{J}_{k}[\mathbf{6}]$.

Recall from $\S 1$ that a $k$-flat structure on a complex $k$-flat structure $(\xi, \nabla)$ on a manifold $M$ induces a chain map $\nabla_{*}: W O_{k}+\Omega_{\mathbf{F}}^{*} M$ whose induced map in cohomology depends only on the concordance class of $(\xi, \nabla)$. Let $\nabla_{*}$ also denote its own restriction to $I_{k} . \nabla_{*}$ induces a map $\Pi \nabla_{*}: I_{k} \rightarrow \Pi^{*} \Omega_{\mathrm{F}}^{*} M$, and the following theorem corresponds to Theorem 3.1 of [6]:

THEOREM 4.6. The map $\Pi \nabla_{*}: \Pi^{*} I_{k} \rightarrow \Pi^{*} \Omega_{\mathrm{F}}^{*} M$ depends only on the concordance class of $(\xi, \nabla)$.

Proof. Let $\left(\xi_{j}, \nabla_{j}\right) \in F_{k}(M \times I)$ and suppose $(\gamma, \hat{\nabla}) \in F_{k}(M \times I)$ is a concordance between $\left(\xi_{0}, \nabla_{0}\right)$ and $\left(\xi_{1}, \nabla_{1}\right)$. The truncated Chern-Weil map $\hat{\nabla}_{*}: I_{k} \rightarrow$ $\Omega^{*}(M \times I)$ satisfies $i_{j}^{*} \circ \hat{\nabla}_{*}=\nabla_{j *}$ for $j=0,1$. Consider the diagram

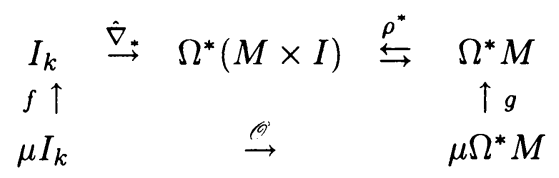

where $f: \mu I_{k}$ and $g: \mu \Omega^{*} M \rightarrow \Omega^{*} M$ are minimal models. By 4.2(i), we can find $\mathscr{O}: \mu I_{k} \rightarrow \mu \Omega^{*} M$ such that the diagram homotopy commutes. Therefore $g \cdot \mathscr{O}=$ $i_{j}^{*} \circ \rho^{*} \circ g \circ \mathscr{O} \simeq i_{1}^{*} \circ \hat{\nabla}_{*} \circ f$ or $\nabla_{0}^{*} \circ f \simeq \nabla_{1}^{*} \circ f$.

REMARK 4.7. (i) The proof of Theorem 4.6 is virtually identical to [6, Theorem 3.1].

(ii) Suppose $f: M \rightarrow N$ is a smooth map and $(\xi, \nabla) \in F_{k}(N)$. Then the following diagram commutes precisely:

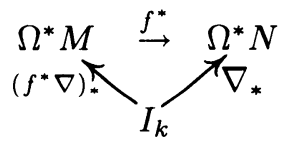

Thus, $\Pi \nabla_{*} \cdot \Pi f^{*} \circ \Pi\left(f^{*} \nabla\right)_{*}: \Pi^{*} I_{k} \rightarrow \Pi^{*} \Omega^{*} N$.

THEOREM 4.8. (i) There are injective maps from $\pi^{*} J_{k} \rightarrow \pi^{*} B C^{k}$ and $\pi^{*} I_{k} \rightarrow$ $\pi^{*} B C_{\mathbf{C}}^{k}$.

(ii) If $(\xi, \nabla)$ is a $k$-flat structure and $f: M \rightarrow B C^{k}$ is its classifying map as in 3.5 , then the diagram

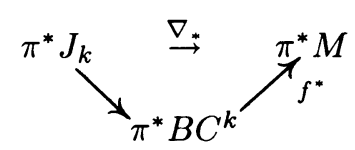

commutes. The analogous statement holds for complex $k$-flat structures.

Proof. Let $\rho: B S C^{k} \rightarrow B C^{k}$ be the universal covering space of $B C^{k}$. We identify $B S C^{k}$ with the pullback of $B C_{k}$ over the covering map $\tilde{\rho}: B S O^{k} \rightarrow B O^{k}$. For any $k$, let $B S O_{n}^{(k)}$ denote the $n$-skeleton of $B S O^{k}$. 
First suppose $k=2 r+1$. By Proposition 3.6 the inclusion $\tilde{j}: B S O_{k}^{(2 k)} \rightarrow B S O_{k}$ lifts as shown:

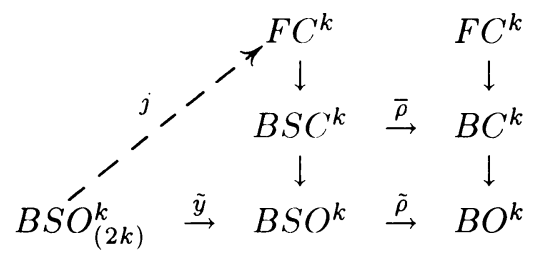

Choose a homotopy equivalence $h: \mathscr{U} \rightarrow B S O_{(2 k)}^{k}$ where $\mathscr{U}$ is an open manifold. By Theorem 3.5, $\rho \circ j \circ h$ classifies (up to homotopy) a $k$-flat structure $(\xi, \nabla)$ on $\mathscr{U}$. Let $\nabla_{*}: I_{k} \rightarrow \Omega_{\mathbf{R}}^{*} \mathscr{U}$ be the induced map and let $\nabla_{*}$ also denote its own restriction to $J_{k}$. By construction,

$$
\nabla_{*}: H^{*} J_{k} \rightarrow P_{\mathbf{R}}^{2 k}\left(C_{1}, \ldots, C_{r}\right) \rightarrow H^{*} \mathscr{U}^{\left(h^{*}\right)^{-1}} H^{*} B S O_{(2 k)}^{k}
$$

is an isomorphism. It now follows from Lemma 4.2 that $\Pi \nabla_{*}: \Pi^{*} J_{k} \rightarrow \Pi^{*} \mathscr{U} \cong$ $\Pi^{*} B S O_{(2 k)}^{k}$ is an isomorphism as well. The universal properties of $B S C^{k}$ together with Remark 4.7 imply that $\Pi^{*} J_{k}$ injects into $\Pi^{*} B S C^{k}$ as well.

Since $\rho^{*}: \Pi^{*} B C^{k} \rightarrow \Pi^{*} B S C^{k}$ is an isomorphism in dimensions $\geq 2$, it follows that $\Pi^{*} J_{k}$ also injects into $\Pi^{*} B C^{k}$.

If $k=2 r$, we replace $B S O_{(2 k)}^{k}$ with $B S O_{(2 k)}^{k}$ and argue as above. In this case, $\Phi_{*}: H^{*} J_{k} \cong P^{2 k}\left(C_{1}, \ldots, C_{n}\right) \rightarrow H^{*} B S O_{(2 k)}^{k-1}$ is an isomorphism.

To prove (ii), suppose we are given $(\xi, \nabla) \in F_{k}(M)$ and its classifying map $f: M \rightarrow B C^{k}$. We will assume $\xi$ is oriented. Then $f$ lifts to a map $\tilde{f}: M \rightarrow$ $B S C^{k}$. Since $M$ is finite dimensional, we can assume, up to homotopy, that $\tilde{f}(M)$ is contained in a finite dimensional subcomplex $K \subset B S C^{k}$. We will also suppose that the $2 k$ skeleton of $B S C^{k}$ is contained in $K$. Choose open manifolds $U, V$, homotopy equivalences $l: K \rightarrow V \cdot j: B S C_{(2 k)}^{k} \rightarrow U$, and a smooth map $g: U \rightarrow V$ homotopic to $l \circ i \circ j^{-1}$ where $i: B S C_{(2 k)}^{k} \rightarrow K$ is the inclusion.

Consider the homotopy commutative diagram

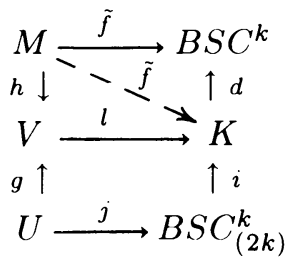

The maps $d \circ l: V \rightarrow B S C^{k}$ and $d \circ i \circ j: U \rightarrow B S C^{k}$ classify $k$-flat structures $\left(\xi_{1}, \nabla_{1}\right) \in F_{k}(U),\left(\xi_{2}, \nabla_{2}\right) \in F_{k}(V)$ such that the diagram

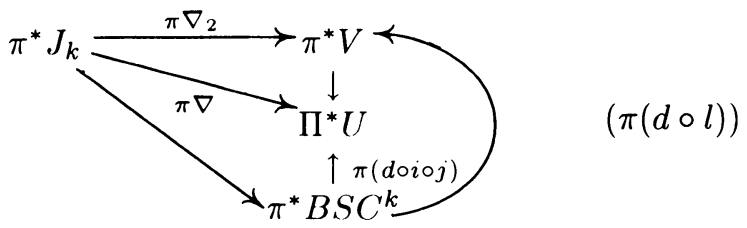


commutes. $(\xi, \nabla)$ is concordant to $h^{*}\left(\xi_{1}, \nabla_{1}\right)$ in $F_{k}(U)$. Hence the diagram

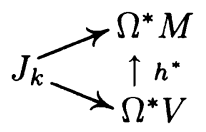

commutes up to chain homotopy, and

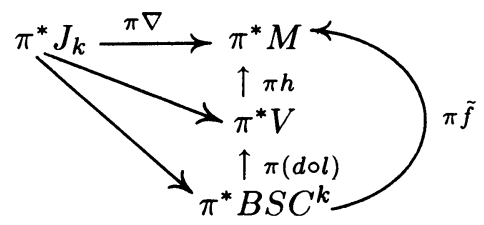

commutes precisely.

The complex case works just like the real case with the simplification that $B U^{k}$ and $B C^{k}$ are simply connected.

Let $B U_{(2 k)}^{k}=2 k$-skeleton of $B U^{k}$. As before, the inclusion $\tilde{j}: B U_{(2 k)}^{k} \rightarrow B U^{k}$ lifts

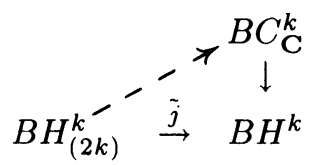

by 3.9 (ii), and an argument identical to the one just used proves $\Pi^{*} I_{k}$ injects into $\Pi^{*} B C_{\mathbf{C}}^{k}$.

COROLLARY 4.9. There are injections $\Pi^{i} J_{k} \rightarrow \Pi^{i} F C^{k}$ and $\Pi^{i} J_{k} \rightarrow$ $\Pi^{i} F C_{\mathbf{C}}^{k}$ in dimensions $i>2 k$.

PROOF. There is a long exact sequence in homotopy

$$
\cdots \Pi^{i} B O^{k} \stackrel{\rho^{*}}{\rightarrow} \Pi^{i} B C^{k} \stackrel{l^{*}}{\rightarrow} \Pi^{i} F C^{k} \stackrel{\delta}{\rightarrow} \Pi^{i+1} B O^{k} \rightarrow \cdots .
$$

According to Theorem 4.4, $\Pi^{i} B O^{k}=0$ if $i>2 k$. Therefore, $l^{*}: \Pi^{1} B C_{k} \rightarrow \Pi^{1} F C_{k}$ is an isomorphism in these dimensions.

The complex case is similar.

We will translate Corollary 4.9 into an equivalent statement about the cohomology of $F C^{k}$ and $F C_{\mathbf{C}}^{k}$.

THEOREM 4.10. There are injections in cohomology $H^{*} V_{k} \rightarrow H^{*} F C_{k}$ and $H^{*} W_{k} \rightarrow H^{*} F C_{\mathbf{C}}^{k}$.

Proof. Let $\mathscr{H}$ denote the dual rational Hurewicz map, and $\overline{\mathscr{H}}$, the algebraic dual Hurewicz map [4].

According to [6], given any foliated manifold $(M, \mathscr{T})$ with trivial normal bundle and adapted connection $\nabla_{\mathscr{J}}$, there is a commutative diagram

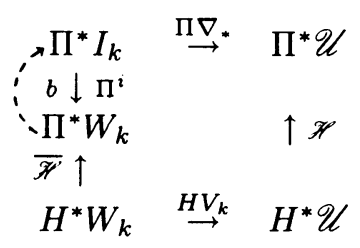


where $b: \Pi^{*} W_{k} \rightarrow \Pi^{*} I_{k}$ is a map of graded vector spaces such that $b \circ \overline{\mathscr{H}}: H^{*} W_{k}$ $\rightarrow \Pi^{*} I_{k}$ is injective and $\Pi i \circ b=\mathrm{id}$. The proof of this theorem extends verbatim to the case of $k$-flat structures and the diagram restricts as follows:

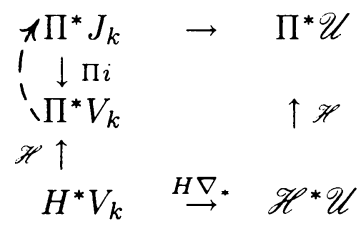

By Corollary 4.9, we have an injection $\Pi^{i}: J_{k} \rightarrow \Pi^{i} F C^{k}$ in dimensions $i>2 k$. Choose a basis of generators $\alpha_{1}, \ldots, \alpha_{n}$ for the cohomology $H^{*} V_{k}$, and a map $f_{i}: S^{\gamma_{i}} \rightarrow F C^{k}$ detecting the image of $\alpha_{i}$ under the map

$$
H^{*} V_{k} \rightarrow \Pi^{*} V_{k} \rightarrow \Pi^{*} J_{k} \rightarrow \Pi^{*} F C^{k}
$$

Let $S=\bigvee_{i=1} S^{\gamma_{i}}, f=\bigvee_{i=1} f^{\gamma_{i}}: S \rightarrow F C^{k}$ and let $h: \mathscr{U} \rightarrow S$ be a homotopy equivalence where $\mathscr{U}$ is an open manifold.

$f \circ h: \mathscr{U} \rightarrow F C_{k}$ classifies a trivial $k$-flat structure $(\xi, \nabla)$ on $\mathscr{U}$, and in the diagrams above, $\Pi \nabla_{k} \circ b \circ \overline{\mathscr{H}}$ is injective by construction. Thus, $\mathscr{H} \circ H \nabla_{*}$ must also be 1-1, and the cohomology $H^{*} V_{k}$ injects into the cohomology of $\mathscr{U}$. The universal properties of $F C_{k}$ now imply that $H^{*} V_{k}$ injects into $H^{*} F C^{k}$.

The complex case is similar.

5. The results in the previous section may be summarized as follows:

1. The exotic characterstic classes $H^{*} W_{k}$ are contained in the cohomology of the classifying space $F C_{\mathbf{C}}^{k}$ for trivialized complex $k$-flat structures.

2. The subset $H^{*} V_{k}$ of $H^{*} W_{k}$ of exotic classes is contained in the cohomology of the classifying spaces for $F C^{k}$ for trivialized real $k$-flat structures. (It is not known whether all of $H^{*} W_{k}$ is contained in $H^{*} F C^{k}$.)

This means in particular that for each $\alpha \in H^{*} W_{k}$, there is a manifold $M$ and a $k$-flat connection $\nabla$ on $M \times R^{k}$ such that $\nabla_{*}(\alpha) \neq 0$ in $H^{*} M$. We outline below some open questions and methods for generating examples.

Foliations. Recall from the Introduction and $\S 1$ that the adapted connection $\nabla$ on the normal bundle to a foliation is $k$-flat. Hence, the universal $k$-flat structure induces maps $O_{1}: B \Gamma_{k} \rightarrow B C^{k}$ and $O_{2}: F \Gamma_{k} \rightarrow F C^{k}$. We would like to know the image of $O_{2 *} H^{*} F C^{k} \rightarrow H^{*} F \Gamma_{k}$.

Discrete groups I. Let $R^{k} \rightarrow E \stackrel{\pi}{\rightarrow} M$ be a vector bundle whose structure group can be reduced to a discrete subgroup $G \leq G l(R, k)$. Then $E$ carries a codimension $k$ foliation of transverse to the fibres of $\pi$. The adapted connection $\Pi$ to the normal bundle of the foliation induces a map $\nabla: E \rightarrow B C^{k}$. Hence precomposition with the zero section $s$ to $\Pi$ induces a map $\nabla \circ s: M \rightarrow B C^{k}$. Since this construction is natural, the universality of $B G$ provides maps $\phi_{1}: B G \rightarrow B C^{k}$ and $\phi_{2}: F G \rightarrow$ $F C^{k}$. As above, we ask: what is the image $\phi_{2}^{*}\left(W_{k}\right) \Pi H^{*} F C^{k}$ ?

Discrete groups II. We describe a second way to generate $k$-flat structures from discrete groups.

Let $M$ be a smooth compact $k$-manifold, $C^{\infty}(M)=$ smooth functions on $M$ with the discrete topology, $G=G l_{n}\left(C^{\infty}(M)\right)$. $G$ is the group of trivializations of the bundle $R^{n} \rightarrow M \times R^{n} \rightarrow M$. Therefore, a $G$-structure on a manifold $W$ (i.e. a map $W \rightarrow B C$ ) determines a principal $G$-bundle $\xi: G \rightarrow X \stackrel{\pi}{\rightarrow} W$. 
Let $\left(U_{\alpha}, \phi_{\alpha}\right)$ be a trivialization of $\xi$, and define $E=\bigcup_{\alpha} U_{\alpha} \times M \times R^{n} \mid \sim$ where $(w, m, v) \in U_{\alpha} \times M \times R^{n} \sim\left(w, m, \phi_{\beta}^{-1} \circ \phi_{\alpha}(w)(m)(v)\right) \in U_{\beta} \times M \times R^{n}$ whenever $w \in U_{\alpha} \wedge U_{\beta}$.

$E$ is an $R^{n}$ vector bundle over $W \times M$ whose restriction to the $W$ factor is flat (since $G$ has the discrete topology). Hence, $E$ is $k$-flat.

The above construction determines a map $\phi: B G \rightarrow B C^{k}$, and it is a fundamental problem to determine how much of the cohomology of $B C^{k}$ can be detected by such cycles. See [18].

\section{REFERENCES}

1. Raoul Bott, Lectures on characteristic classes and foliations, Notes by Lawrence Conlon, Mexico, 1971, Lecture Notes in Math., vol. 279, Springer, New York, 1973.

2. __ On a topological obstruction to integrability, Proc. Sympos. Pure Math., vol. 16, Amer. Math. Soc., Providence, R.I., 1970, pp. 127-131.

3. R. Bott and J. Heitsch, A remark on the integral cohomology of $B \Gamma q$, Topology 11 (1972), $141-146$.

4. A. K. Bousfield and V. K. A. M. Gugenheim, On PL De Rham theory and rational homotopy type, Mem. Amer. Math. Soc., vol. 179, 1976.

5. André Haefliger, Homotopy and integrability, Manifolds, Amsterdam 1970, Lecture Notes in Math., vol. 209, Springer-Verlag, Berlin, 1971, pp. 128-141.

6. Steven E. Hurder, Dual homotopy invariants of $G$-foliations, Topology 20 (1981), 365-387.

7. John N. Mather, Integrability in codimension one, Comment. Math. Helv. 48 (1973), 195-223.

8. Peter May, Simplicial objects in algebraic topology, Van Nostrand, 1969.

9. John Milnor and James D. Stasheff, Characteristic classes, Princeton Univ. Press, Princeton, N.J., 1974.

10. C. P. Rourke and B. J. Sanderson, $\Delta$-sets I: Homotopy theory, Quart. J. Math. 22 (1971), 321-338.

11. D. Sullivan, Infinitesimal computations in topology, Inst. Hautes Études Sci. Publ. Math. 47 (1977), 269-332.

12. William Thurston, The theory of foliations of codimensions greater than one, Comment. Math. Helv. 49 (1974), 214-231.

13. __ Existence of codimension one foliations, Ann. of Math. 104 (1976), 249-268.

14. James R. Munkres, Elementary differential topology, Princeton Univ. Press, Princeton, N.J., 1963.

Graduate School and University Center (CUNy), 33 West 42 Street, New YORK, NEW YORK 10036 8099

Current address: Department of Mathematics, Brooklyn College, Brooklyn, New York 11210 